Article

\title{
Impact of the Built Environment Measured at Multiple Levels on Nonmotorized Travel Behavior: An Ecological Approach to a Florida Case Study
}

\author{
Jina Mahmoudi * and Lei Zhang \\ Maryland Transportation Institute, University of Maryland, College Park, MD 20742, USA; lei@umd.edu \\ * Correspondence: zhina@umd.edu
}

Received: 30 August 2020; Accepted: 21 October 2020; Published: 24 October 2020

check for updates

\begin{abstract}
Research continues to reveal the benefits of nonmotorized travel modes such as walking and bicycling. Therefore, identification of the factors that nurture these activities is essential in developing sustainable urban planning policies and designs. Among those factors are the built environment characteristics of the place of residence. To date, research on the role of the built environment in nonmotorized travel has focused on neighborhood-level factors. However, people do not stay within their neighborhoods; they live and work at a regional scale and travel to various destinations and distances each day. Nonetheless, little is known about the impact of built environment factors at larger spatial scales on nonmotorized travel behavior. Guided by the principles of the ecological model of behavior, this study investigates the role of the built environment at hierarchical spatial scales in nonmotorized travel behavior. Multilevel Structural Equation Models have been developed to comprehensively examine the complex links between the built environment and individuals' nonmotorized travel. Findings indicate that built environment factors at multiple spatial scales can influence nonmotorized travel behavior. Thus, to promote walking and bicycling, more effective policies are those that include multilevel built environment and land use interventions and consider the overall physical form of urban areas.
\end{abstract}

Keywords: nonmotorized travel behavior; walking; bicycling; built environment; ecological model

\section{Introduction}

Statistics and research findings pertaining to the state of travel within the U.S. reveal that the automobile remains the dominant mode of transportation in the U.S.-particularly for commuting [1-4], whereas walking and bicycling remain the modes less traveled. The most recent National Household Travel Survey (NHTS) showed that of all trips taken in the U.S., the shares of walking and bicycling trips were approximately $11 \%$ and $1 \%$, respectively, compared to the share of trips made by private automobiles, which accounted for nearly $83 \%$ [4].

These trends have adverse health, economic, and environmental consequences. For instance, by 2016 , approximately $30 \%$ of the U.S. population was obese, over $31 \%$ lived with high blood pressure, and nearly 10\% suffered from diabetes [5]. Other statistics reveal that between 2000 and 2014, the economic cost of congestion in urban areas increased by $40 \%$ due to the average commuter wasting 19 gallons of fuel in 2014 as a result of congestion, and by 2016, the transportation sector accounted for approximately $70 \%$ of the total petroleum consumed in the U.S. [6]. Further, according to the U.S. Environmental Protection Agency (EPA), transportation activities accounted for approximately $35 \%$ of U.S. carbon dioxide $\left(\mathrm{CO}_{2}\right)$ emissions from fossil fuel combustion, and $27 \%$ of total U.S. greenhouse gas emissions in 2015 [7].

On the other hand, research continues to reveal health, economic, and environmental benefits of nonmotorized travel—both for individuals and communities. Walking and bicycling are deemed 
sustainable, cost-effective, and environmental-friendly modes of travel with a potential to lower the risk of many health problems including obesity, chronic diseases, mental disorders, and mortality [8-12]. Considering the numerous benefits of walking and bicycling, promoting these nonmotorized modes of travel has become the focus of many transportation and planning professionals and agencies in recent years. As a result, land use policies that encourage moving away from a sedentary lifestyle (in which automobile is the dominant mode of travel) and moving toward more sustainable travel patterns and a more viable lifestyle (in which nonmotorized travel modes are utilized more) are gaining increased importance.

Within a sustainable transportation context, identification of factors that influence nonmotorized travel behavior and understanding the extent and direction of the effects of those factors are essential to the development of travel demand models with a comprehensive, multimodal framework. The built environment characteristics of the residential location are among the many factors that can influence the choice and the extent of nonmotorized travel by individuals.

The built environment can be measured at various spatial scales including at the block level, the neighborhood level, the county level, and the metropolitan area level. These spatial scales can be accordingly categorized into hierarchical levels of the built environment such as (1) the micro-level built environment, which represents the built environment characteristics of the block or neighborhood; (2) the meso-level built environment, which represents the built environment characteristics of the region or county; and (3) the macro-level built environment, which represents the built environment characteristics of the metropolitan area. This spatial categorization of the built environment is in line with the three-level (i.e., micro-meso-macro) spatial hierarchy for the built environment suggested in previous research $[13,14]$.

While it is evident from past research findings that micro-level (e.g., neighborhood-level) built environment factors influence nonmotorized trips [15,16], it is not clear how the built environment characteristics of higher-level spatial areas affect such trips. The underlying reason for this lies in the assumption that most nonmotorized travel is spatially constrained, and therefore, smaller spatial units of analysis (e.g., neighborhood) yield more information on the built environment characteristics that impact nonmotorized trips. Theoretically, however, there are reasons to think beyond the neighborhood boundaries in conducting nonmotorized travel behavior research:

First, from a travel behavior standpoint, the location of the neighborhood should be considered in the context of the surrounding area's built environment such as that of the metropolitan area. For instance, if the overall built environment of the metropolitan area (i.e., the macro-level built environment) is heavily car-dependent, the walkable/bikeable design of a particular neighborhood (i.e., the micro-level built environment) may not matter much in promoting the walking/bicycling mode choices within that neighborhood. That is, islands of walkable/bikeable neighborhoods surrounded by typical car-dependent, low-density, suburban neighborhoods in a sprawled metropolitan area may not lead to fundamental changes in the overall travel behavior of residents of those walkable/bikeable neighborhoods $[17,18]$. Further, clusters with just one feature of walkable/bikeable neighborhood design may not change travel behavior in favor of nonmotorized travel. For example, a dense residential-only neighborhood far from nonresidential services does little in promoting nonmotorized trips due to distances between residences and land uses, just as a neighborhood with a mix of land uses may not promote nonmotorized trips if it is surrounded by high-speed highways [19]. Therefore, larger spatial contexts bear importance in shaping people's travel behavior-including their nonmotorized travel choices-and should be considered in the analysis of nonmotorized travel behavior.

Moreover, findings of previous research show that metropolitan area-level (i.e., macro-level) built environment influences vehicular travel behavior [20]. Therefore, it can be hypothesized that the overall built environment of the metropolitan area has a potential to impact nonmotorized travel behavior - at least through its impact on motorized travel behavior. Particularly, urban sprawl and decentralization are important metropolitan area-level built environment factors with the potential to influence nonmotorized travel behavior. These features lead to increased trip distances, which in turn, 
can promote private vehicle use and discourage nonmotorized modes of travel by making them unsafe or impractical. Macro-level built environment features such as sprawling suburban developments have been suggested to discourage nonmotorized travel choices such as walking [21]. One reason can be that urban sprawl and decentralization usually produce long commutes for residents. Sprawling metropolitan areas with longer commutes can affect recreational walking (or bicycling) by cutting leisure times short [22]. In light of these arguments, consideration of measures representing the level of sprawl within the metropolitan area in the analysis of nonmotorized travel behavior seems a plausible idea.

Additionally, existing travel behavior literature hints at the potential role of the built environment at larger spatial scales in nonmotorized travel behavior. One study postulated that the built environment—at many spatial scales including the region—can affect the propensity of being physically active (e.g., engage in nonmotorized travel) and characterized the lack of consideration of the built environment at scales larger than the neighborhood as a "notable gap" in nonmotorized travel behavior research [1]. It has also been argued that because people spend most of their daily hours away from their home, the implicit assumption that walking distance from place of residence is the operative scale at which the built environment influences physical activity (e.g., nonmotorized travel) is just that: "an assumption" [22].

Therefore, it becomes evident that travel behavior is affected by opportunities and constraints at multiple levels of influence, as suggested by Van Acker et al. [23], and the attractiveness of a location (e.g., travel destination) depends not only on its immediate neighborhood but also on how it spatially relates to the urban area it locates in, as suggested by Guo and Bhat [24]. These notes in travel behavior literature provide grounds for the hypothesis that nonmotorized travel behavior is not just influenced by micro-level built environment characteristics but also by built environment characteristics of larger spatial areas. This means that in influencing nonmotorized travel behavior, neighborhoods do not act as isolated entities but as an interwoven part of the larger spatial context of the region and metropolitan area they belong to.

Second, from a human behavior standpoint, behavioral theories such as the ecological model of behavior suggest that any activity or behavior results from the influences of and the interaction between multiple levels of the social as well as the built environment [25]. This implies that, like any human behavior, walking and bicycling activities can also be influenced by factors representing multiple spatial levels of the built environment including those representing areas beyond the neighborhood.

In fact, a review of the theoretical foundations for human behavior-which mostly come from the field of psychology-reveals that the chief theory that can explain the role of the built environment in nonmotorized travel behavior is the ecological model of behavior. The framework for the ecological model of behavior incorporates all the components of the theory of planned behavior (i.e., attitudes, subjective norms, and perceived behavioral controls) [26] as well as those of the social cognitive theory (i.e., social environment factors) [27] and adds to them the influence of the built environment factors on behavior. The ecological model framework can include factors representing hierarchical spatial levels of the built environment such as the micro level (e.g., the neighborhood), the meso level (e.g., the county), and the macro level (e.g., the metropolitan area or city). With a central focus on the role of the built environment at multiple spatial levels in human behavior, the ecological model framework can be considered the most integrated and suitable theoretical framework for analysis of the link between nonmotorized travel behavior and the built environment.

The above theoretical arguments from the travel behavior and the human behavior research fields elucidate the complex interrelation between the built environment and travel behavior and justify testing the potential impact of the built environment at larger spatial levels on nonmotorized travel behavior. More specifically, in becomes clear that to systematically test the mechanisms behind the built environment and nonmotorized travel connection, it is important to sketch out a comprehensive framework that includes built environment characteristics at multiple spatial levels. 
Therefore, in examining the link between the built environment and nonmotorized travel behavior, a more comprehensive analysis is one that considers the built environment at larger spatial levels (e.g., the meso- and the macro-level built environments) in addition to the micro-level built environment. Such empirical analysis could give a more complete picture of factors influencing individuals' nonmotorized travel behavior and its link with the built environment attributes of their place of residence. Nonetheless, empirical studies on the role of the built environment characteristics beyond the neighborhood boundaries in nonmotorized travel behavior remain scant. This gap in knowledge highlights the need for research into the role of regional (i.e., meso-level) and metropolitan area (i.e., macro-level) built environment factors in promotion or prevention of nonmotorized trips.

Considering the above arguments, the present study aims to probe the link between nonmotorized travel behavior and multiple spatial levels of the built environment. Structural Equations Modeling (SEM) techniques have been employed to account for the effects of residential self-selection in the analysis. The main research hypotheses are:

- In addition to those from the neighborhood (i.e., the micro level), built environment factors from larger spatial levels such as those representing the county (i.e., the meso level) and the metropolitan area (i.e., the macro level) play a role in nonmotorized travel behavior;

- Residential self-selection plays a role in nonmotorized travel behavior; and

- The link between nonmotorized travel behavior and built environment factors-as measured in the sample analyzed-is a causal one.

Investigating the effects of the built environment at different spatial levels including the neighborhood, the county, and the metropolitan area on nonmotorized travel behavior is a research idea that fits the framework of the ecological model of behavior but has not been previously explored. The present study contributes to the existing empirical knowledge on the link between nonmotorized travel behavior and the built environment by using an ecological framework to examine the effects of built environment factors at multiple spatial levels on nonmotorized trips. As the influence of the built environment beyond the neighborhood level on nonmotorized travel behavior is yet to be determined, the simultaneous inclusion of various spatial levels of the built environment-as done in this study-sheds light on the influence exerted by factors at each level. This can assist transportation/urban planners and policy decision-makers in identification of the most promising interventions that target the built environment and development of more effective policies through which more sustainable modes of travel are promoted.

\section{Literature Review}

\subsection{The Role of Sociodemographic and Socioeconomic Factors in Nonmotorized Travel Behavior}

Empirical studies on nonmotorized travel behavior provide evidence that sociodemographic and socioeconomic characteristics including age, gender, race, car ownership, and income influence walking and bicycling trips. Age appears to have a negative correlation with nonmotorized travel based on previous findings, e.g., [28,29]. With respect to gender, past studies suggest that higher levels of nonmotorized travel are generally associated with being male [16,28-32]. Race seems to have a significant association with walking and bicycling with many studies suggesting that non-white individuals are more likely to make nonmotorized trips [30,33-35]. Past studies also suggest that higher income correlates with lower levels of nonmotorized travel, e.g., [3,35]. However, the findings on income are not universal and vary based on the context or trip purpose [30,31]. Moreover, access to a private vehicle is almost always negatively associated with walking and bicycling in the past studies $[16,30,32,35-37]$. 


\subsection{The Role of Built Environment Factors in Nonmotorized Travel Behavior}

Five key built environment dimensions that have a potential to influence nonmotorized travel behavior can be derived from the evidence provided by previous research. These include: density, diversity, design, destination accessibility, and distance to transit. These dimensions are referred to as the five Ds of the built environment [38].

Density has been operationalized in various studies using various methods yielding the use of different measures of density such as population density, employment density, residential density, housing density, and activity density, e.g., [39-41]. Regardless of the density measure used, existing literature suggests that the effects of neighborhood-level density on nonmotorized travel behavior remain unclear. This statement is consistent with previous literature arguing that compared to other $D$ s of the built environment, density is of less importance and its effects sometimes are captured by the other Ds [38]. Previous findings on the association between the extent of neighborhood diversity (i.e., mixed land use) and nonmotorized travel are also inconsistent. Many studies found that presence of or proximity to mixed-use and/or commercial establishments was positively associated with nonmotorized travel, e.g., [30,31,33,42]. However, others reported mixed, negative, or non-existent correlations between local mixed land use or commercial establishments and nonmotorized travel, e.g., $[16,31,43]$. Moreover, findings of previous studies suggest that the design characteristics of the neighborhood such as block size, extent of street connectivity, and density of intersections play a role in nonmotorized travel behavior, e.g., [40,43]. Past research suggests that local destination accessibility also plays a role in nonmotorized travel behavior. Particularly, limited access to local destinations in terms of distance (i.e., greater distances to destinations) has been reported in many studies to be negatively associated with nonmotorized travel $[1,15,30,42,44-46]$. Findings of the studies that examined the role of distance to transit facilities on walking and bicycling suggest that shorter distances to local transit are correlated with higher rates of nonmotorized travel, e.g., [47-49].

A notable knowledge gap revealed from the literature review is the inattention of existing empirical research efforts to the effects of various levels of geography on nonmotorized travel behavior. To date, almost all the empirical studies that examined the association between nonmotorized travel behavior and the built environment focused on the micro-level (i.e., neighborhood-level) built environment factors, and did not include built environment attributes from larger levels of geographical aggregation. Some researchers have suggested that macro-level built environment characteristics such as regional accessibility, urban sprawl, and decentralization have a potential to affect walking and bicycling travel by promoting sedentary behavior and increasing automobile use $[1,13,50]$. Nonetheless, empirical studies that considered the role of macro-level built environment in nonmotorized travel behavior are scarce. Findings of these few studies indicated that: (i) regional (zip code level) densities did not impact walking [15]; (ii) metropolitan area-level compactness promoted walking [14]; and (iii) regional-level walkability influenced walking mode share [41]. With regards to bicycling, past empirical findings showed that more city-level bicycle infrastructure was associated with higher rates of bicycle commuting [36].

\subsection{The Role of Psychological Factors in Nonmotorized Travel Behavior}

Literature provides evidence that psychological factors such as attitudes, perceptions, and preferences play key roles in nonmotorized travel behavior [35,37,45,51]. Findings of these studies suggest that pro-walking, pro-bicycling, pro-environment, and pro-health individuals are more likely to choose nonmotorized modes of travel. Some of these studies argue that the effects of attitudinal predispositions may supersede the effects that built environment and land use factors exert on nonmotorized mode choices, e.g., [42,51]. Literature further suggests that attitudes and preferences also influence residential location choices-leading to self-selection bias. That is, individuals with a preference toward nonmotorized modes of travel may self-select themselves into pedestrian- and bicyclist-friendly residential areas. In this case, individuals' residential location choices-and not the 
effect of built environment attributes—can be considered as a possible explanation for nonmotorized travel occurring at those locations [42,45,52].

Due to the concurrent influence of attitudes on both individuals' travel choices and residential location choices, self-selection may result in spuriousness [21,44] —an effect that confounds the relationship between nonmotorized travel behavior and the built environment. More specifically, the self-selection argument addresses the issue of correlation or causality. Here, the argument is that existence of a correlation between built environment characteristics and nonmotorized travel behavior does not guarantee existence of a causal link between the two [52]. Some researchers suggest that in examining the link between the built environment and travel behavior such as nonmotorized travel behavior, residential self-selection should be controlled for because otherwise, the analysis may produce biased results $[1,53]$. Among the empirical studies that did address self-selection bias, many found a statistically significant influence for built environment attributes on nonmotorized travel behavior, even after accounting for residential self-selection [15,42,53-55]. To circumvent self-selection bias, various methodologies have been used in previous research including: direct surveys [55], propensity score analysis [53], instrumental variable analysis [15], and structural equation modeling [20,43]. Among these, the structural equation modeling methodology emerges from the existing literature as a prominent approach to account for self-selection bias in examining the link between travel behavior and built environment characteristics. By allowing multiple directions of causality, the structural equation modeling methodology offers a conceptual improvement over the single-equation methodology (i.e., ordinary least square methodology) [21].

Literature also suggests that psychological factors related to the social environment of residence influence nonmotorized travel. Particularly, factors representing social and cultural norms, social values, public image, and prestige appear to impact nonmotorized travel $[28,40,45,56,57]$. The effects of psychological concepts such as observational learning [58] and contagion perspectives [29] on nonmotorized trips are of particular interest. Moreover, the social and cultural norms within the community of prior residence can influence nonmotorized travel behavior of individuals. This effect can be considered from an immigration perspective. Past research found that living in areas with a high population of immigrants was linked with higher levels of walking to school among children [57], whereas having a U.S.-born parent was associated with a lower likelihood of walking/bicycling to school by children [59]. Evidence is also provided that other social environment factors with a psychological effect can play a role in nonmotorized travel behavior. An example of such factors is crime. Perceptions of crime as well as actual crime rates have been found by some researchers to be negatively associated with nonmotorized travel $[10,29,57,60]$.

\subsection{Application of the Ecological Model of Behavior in Nonmotorized Travel Behavior}

The usage of ecological model of behavior-or extensions of it-has been recommended in previous research to develop conceptual frameworks for examination of nonmotorized travel behavior $[61,62]$. As a result, the ecological model has increasingly been adopted in analysis of walking and bicycling trips in recent years. Among such empirical studies is Wang et al. [63], which used the ecological model to examine the interactions of psychosocial factors (i.e., social environment factors) with neighborhood-level built environment factors in explaining adolescents' nonmotorized travel behavior. They found that built environment factors such as neighborhood walkability as well as psychosocial factors such as social support from peers had significant positive effects on nonmotorized travel [63]. The authors indicated that their findings provided support for the ecological model's principle of interactions across various levels of influence and highlighted the importance of both built environment and psychosocial factors (i.e., social environment factors) in shaping adolescents' nonmotorized travel. Further, Mitra and Nash [64] employed an ecological approach to explore the correlation between various trip-related, sociodemographic, built environment, and attitudinal factors and the likelihood of bicycling as well as gender-based differences in bicycling by university students. A key finding of 
this study was that the built environment correlates of bicycling for commute trips were different from those for non-commute trips, particularly for female students [64].

\section{Data and Methodology}

\subsection{Study Area}

The present study uses data from several metropolitan areas within the state of Florida in the U.S. According to the U.S. Census Bureau, a Core-Based Statistical Area (CBSA) designated as a metropolitan area has "at least one urbanized area of 50,000 or more population, plus adjacent territory that has a high degree of social and economic integration with the core as measured by commuting ties" [65]. For the purpose of this case study, a metropolitan area is considered the CBSA where the surveyed household was located. Data from 23 metropolitan areas (CBSAs) in the state of Florida are utilized in the analysis. Table 1 lists the Florida metropolitan areas used in this study along with their corresponding sample size and total population.

Table 1. Florida case study metropolitan areas.

\begin{tabular}{|c|c|c|c|c|c|}
\hline Metropolitan Area (CBSA) & $\begin{array}{l}\text { Sample } \\
\text { Size }\end{array}$ & $\begin{array}{l}\text { Total Population } \\
\text { (2010) }^{1}\end{array}$ & $\begin{array}{c}\text { Total Employment } \\
\text { (2010) }^{1}\end{array}$ & $\begin{array}{c}\text { Mean Residential } \\
\text { Density (Persons/Acre) }{ }^{2}\end{array}$ & $\begin{array}{c}\text { Mean Employment } \\
\text { Density (Jobs/Acre) }{ }^{2}\end{array}$ \\
\hline Cape Coral-Fort Myers & 478 & 618,754 & 179,096 & 3.62 & 0.92 \\
\hline Crestview-Fort Walton Beach-Destin & 325 & 180,822 & 85,495 & 3.29 & 1.14 \\
\hline Deltona-Daytona Beach-Ormond Beach & 448 & 494,593 & 151,016 & 3.36 & 0.85 \\
\hline Gainesville & 310 & 264,275 & 117,721 & 2.73 & 1.77 \\
\hline Homosassa Springs & 354 & 141,236 & 33,638 & 1.06 & 0.21 \\
\hline Jacksonville & 1184 & $1,345,596$ & 653,161 & 4.27 & 1.41 \\
\hline Lakeland-Winter Haven & 406 & 602,095 & 192,325 & 2.84 & 1.31 \\
\hline Miami-Fort Lauderdale-Pompano Beach & 3949 & $5,564,635$ & $2,118,833$ & 13.54 & 2.81 \\
\hline Naples-Marco Island & 264 & 321,520 & 108,302 & 4.24 & 1.17 \\
\hline North Port-Bradenton-Sarasota & 516 & 702,281 & 219,985 & 4.28 & 1.39 \\
\hline Ocala & 315 & 331,298 & 88,705 & 1.54 & 0.35 \\
\hline Orlando-Kissimmee-Sanford & 1155 & $2,134,411$ & 978,967 & 4.83 & 1.47 \\
\hline Palm Bay-Melbourne-Titusville & 447 & 543,376 & 184,272 & 4.43 & 1.15 \\
\hline Palm Coast & 146 & 95,696 & 19,020 & 2.44 & 0.44 \\
\hline Panama City-Lynn Haven-Panama City Beach & 296 & 168,852 & 75,398 & 2.58 & 1.41 \\
\hline Pensacola-Ferry Pass-Brent & 518 & 448,991 & 192,352 & 2.65 & 0.98 \\
\hline Port St. Lucie & 429 & 424,107 & 114,037 & 3.51 & 0.87 \\
\hline Punta Gorda & 165 & 159,978 & 37,142 & 2.56 & 0.71 \\
\hline Sebastian-Vero Beach & 115 & 138,028 & 44,289 & 2.51 & 0.87 \\
\hline Sebring & 274 & 98,786 & 25,528 & 1.95 & 0.61 \\
\hline Tallahassee & 617 & 367,413 & 184,366 & 2.48 & 1.31 \\
\hline Tampa-St. Petersburg-Clearwater & 2160 & $2,783,243$ & $1,046,561$ & 6.35 & 1.74 \\
\hline The Villages & 105 & 93,420 & 20,675 & 1.94 & 0.28 \\
\hline
\end{tabular}

${ }^{1}$ Source of data: Smart Location Database (SLD); ${ }^{2}$ mean population and employment densities have been computed for each metropolitan area based on information available in the SLD.

As the table indicates, the most highly populated metropolitan areas in the Florida sample are the Miami-Fort Lauderdale-Pompano Beach and the Tampa-St. Petersburg-Clearwater metropolitan areas, respectively. In terms of average employment density, Miami-Fort Lauderdale-Pompano Beach is the metropolitan area with the most job opportunities per area of land, and the Gainesville and the Tampa-St. Petersburg-Clearwater metropolitan areas follow next. Table 1 also shows that sample sizes are proportionate to the population, which overall makes this sample a good representative of the population within the state of Florida.

\subsection{Data}

The database for the nonmotorized travel behavior models developed in this study consists of the following individual datasets:

- American Community Survey (ACS);

- County Health Rankings \& Roadmaps (CHR\&R);

- National Household Travel Survey (NHTS) - 2009 Florida Add-on Program;

- Smart Location Database (SLD);

- Topologically Integrated Geographic Encoding and Referencing (TIGER)/Line Shapefiles; 
- Uniform Crime Reporting (UCR) Program;

- Urban Mobility Information;

- Walk Score.

A brief description of each of these datasets and how they have been used in this study is provided below.

ACS: The American Community Survey (ACS) is an annual survey program conducted by the United States Census Bureau. Data collected through this survey provide information about the population (e.g., socioeconomic and sociodemographic characteristics, means of commuting to work, disability characteristics) as well as housing (e.g., financial and physical characteristics for housing units) at many geographical scales. Data from several multiyear estimates of ACS have been used in this study to develop nonmotorized travel behavior models.

CHRER: The Robert Wood Johnson Foundation in collaboration with the University of Wisconsin Population Health Institute created the County Health Rankings and Roadmaps (CHR \& R) program. The CHR \& R dataset is publicly available and provides information on county-level health profile and rankings for each U.S. county. The CHR \& R data have been used in this study to obtain violent crime rates within the Florida metropolitan areas for which crime rate data were not available through the FBI's UCR program.

NHTS: The National Household Travel Survey (NHTS) dataset is the only comprehensive travel dataset at the national level in the U.S. The survey is periodically conducted by the Federal Highway Administration (FHWA) to collect data on both short- and long-term travel behavior of individuals living in the U.S. The NHTS dataset contains comprehensive information on household geographic area (e.g., census tract, county, and metropolitan area), household socioeconomic and sociodemographic characteristics (e.g., household income, size, number of vehicles, and number of workers), as well as detailed information on respondents' daily trips made within a designated 24-h period of time (e.g., trip mode, travel time, trip purpose, and distance traveled).

As a nationwide travel survey, the NHTS greatly challenges the ability to measure the micro-level built environment near respondents' residences. Instead, the Florida 2009 NHTS Add-on travel survey data identified the census block group of each household as the smallest geographic unit in the survey. This facilitated the operationalization of the micro-level (i.e., neighborhood-level) built environment in the present study. The Add-on data also provided geocoded information on Florida survey respondents' household location, household socioeconomic and sociodemographic characteristics, as well as detailed information on trips made by individuals within each surveyed household. Figure 1 shows the mode share for trips made in Florida based on the 2009 NHTS Add-on travel survey data. Figure 2 shows the percentage of those trips by destination based on the data recorded in the Florida 2009 NHTS Add-on sample.

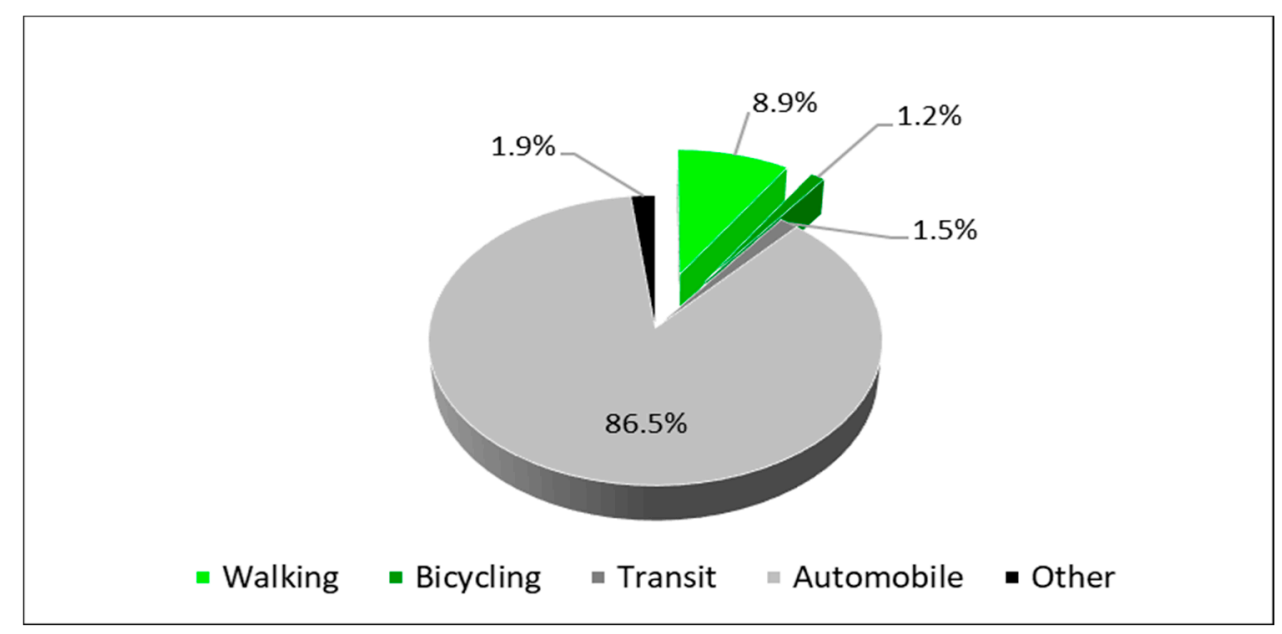

Figure 1. Trip mode share. (source of data: Florida 2009 NHTS Add-on data). 


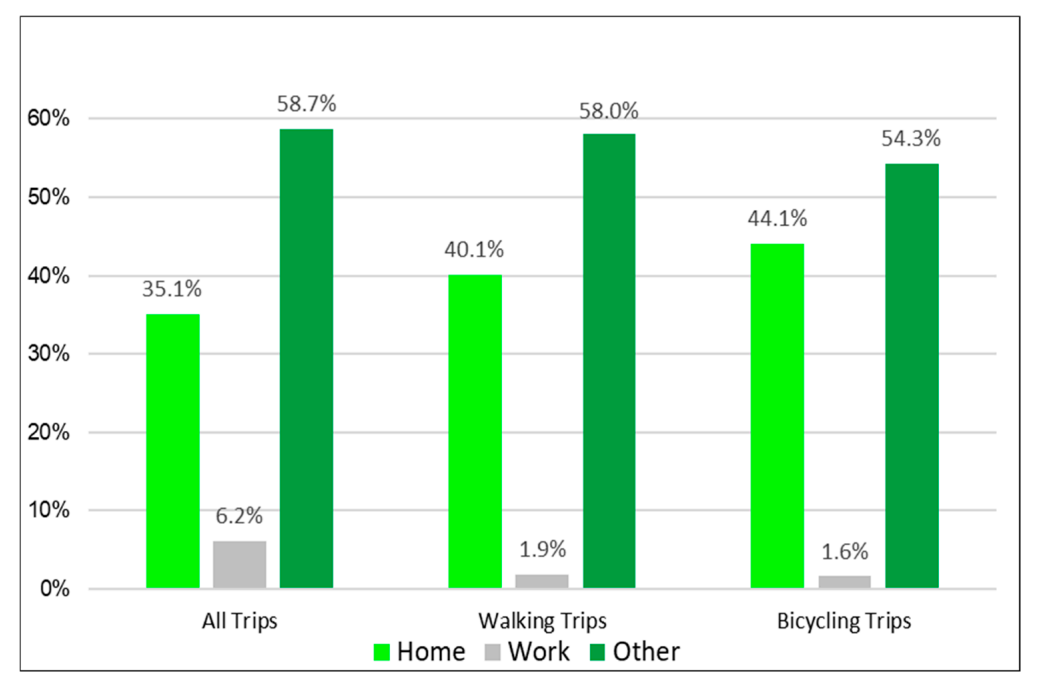

Figure 2. Percentage of trips by destination. (source of data: Florida 2009 NHTS Add-on data).

As it can be seen from Figure 2, "Home" was reported as the destination for over $40 \%$ of the walking trips and over $44 \%$ of bicycling trips in Florida. Considering walking and bicycling trips as one general "nonmotorized" mode, the Florida 2009 NHTS Add-on data also indicate that of the 11,614 nonmotorized trips that were reported in Florida:

- A total of 4708 trips (i.e., approximately $41 \%$ ) were listed with "Home" as trip destination;

- A total of 1635 trips (i.e., over $14 \%$ ) went beyond 1.5 miles in trip distance;

- A total of 1567 trips (i.e., approximately 13.5\%) lasted longer than $30 \mathrm{~min}$ in trip duration.

These statistics show that considerable proportions of Florida walking and bicycling trips did not originate from the residence of the trip-maker and may not have stayed within the neighborhood boundaries.

SLD: The Smart Location Database (SLD) is a nationwide spatial dataset for the U.S. The SLD is a product of the U.S. Environmental Protection Agency (EPA)'s Smart Growth Program and is available to users for free on the EPA web site. This rich dataset provides information on land use and built environment characteristics such as population and employment density, mixed-use development, neighborhood design attributes, destination accessibility, transit accessibility, transit service frequency, and socioeconomic as well as sociodemographic characteristics at the census block group level. In essence, the SLD provides a wealth of information on the $D$ indicators of the built environment at a fine spatial resolution (i.e., census block group). The present study uses the SLD as the source for built environment data in the analysis of nonmotorized travel behavior of Florida residents.

TIGER/Line Shapefiles: The U.S. Census Bureau's TIGER/Line Shapefiles constitute a comprehensive dataset that provides valuable spatial information for use with Geographic Information Systems (GIS) applications. Information such as legal and political geographic boundaries, statistical geographic areas, address information, roads, and railroads can be obtained from this dataset. TIGER/Line Shapefiles are open source data, and in this study, they have been used to obtain census block-level and county-level spatial data within the study area.

UCR: Created in 1929, the FBI's Uniform Crime Reporting (UCR) program is intended to serve the need for reliable crime statistics for the U.S. through collecting, publishing, and archiving data on crime. As of the present, data have been received from more than 18,000 agencies within the U.S. and are available to the public free of charge. This research uses multiyear UCR data on violent crime rates within Florida metropolitan areas in analysis of residents' nonmotorized travel behavior.

Urban Mobility Information: The Texas A\&M Transportation Institute (TTI)'s Urban Mobility Information (referred to as UMI in this study) data are accessible to the public, and provide multiyear data on the level of mobility (e.g., roadway congestion levels, annual hours of delay, travel times) for 
nearly 500 urban areas across the U.S. To develop nonmotorized travel behavior models, the present study uses multiyear UMI data to obtain information on public transportation usage levels, roadway congestion levels, and fuel costs within Florida metropolitan areas.

Walk Score: Walk Score ${ }^{\circledR}$ (www.walkscore.com) is a publicly available dataset that provides information on the walkability of locations. A Walk Score is an objectively measured number that assesses the walkability and pedestrian friendliness of a particular address based on a destination accessibility-oriented approach. Distance to nearby desired walkable amenities is used in an algorithm that calculates the Walk Score of a particular point. Research suggests that Walk Score correlates well with objective and subjective measures of walkability and is a reliable and valid metric for estimating destination accessibility $[66,67]$. Others have also validated Walk Score as a measure of walkability at multiple spatial scales and for various geographic locations [68]. Further, Walk Score has been shown to outperform other measures of walkability in predicting actual amounts of walking [69]. As a result, Walk Score has become a widely known measure of walkability in the past decade and has been used in many studies (see, e.g., [41,70]). Walk Score ranges from 0 for a non-walkable (i.e., car-dependent) location to 100 for the most pedestrian-friendly location. This study utilizes Walk Score data to examine the roles of destination accessibility and walkability in nonmotorized travel behavior of Florida residents.

\subsection{Model Framework}

Applying the ecological model's concepts to walking and bicycling activities, it can be said that:

(i). In examining walking and bicycling behavior, the role of various factors across multiple ecological levels of influence (i.e., intrapersonal, interpersonal, environmental, community, and policy) should be considered;

(ii). Levels of walking or bicycling are expected to be maximized when: (a) the individuals have pro-walking/pro-bicycling attitudes (i.e., representing the influence of the intrapersonal level); (b) the social/cultural norms within the community as well as family and friends are supportive of walking and bicycling (i.e., representing the influences of the interpersonal and the community levels); (c) the built environment-at various spatial scales—and the natural environment are conducive to nonmotorized travel (i.e., representing the influence of the environmental level); and (d) policies are in place to promote walking and bicycling (i.e., representing the influence of the policy level).

The above arguments are also in line with the conceptual model of travel behavior proposed by Van Acker et al. [23], which considered travel behavior as the outcome of a decision hierarchy based on three levels of "opportunities and constraints": (1) individual (i.e., personal sociopsychological characteristics such as attitudes and perceptions); (2) social (i.e., sociodemographic/socioeconomic factors such as age and income, as well as sociocultural characteristics such as ethnicity backgrounds); and (3) spatial (i.e., built/natural environment characteristics) [23].

Therefore, in developing a model framework for analysis of nonmotorized travel behavior, it should be borne in mind that each individual: (a) has certain characteristics including attitudes, age, gender, employment status, etc.; (b) belongs to a social network of family, friends, and colleagues; and (c) resides within a particular location - all of which can drive or impede her/his behavior. Thus, as Van Acker et al. [23] suggests, the ecological model framework should include the individual level, the social environment level, and the spatial (i.e., built) environment level. Accordingly, these three ecological levels have been included in the nonmotorized travel behavior model framework developed in this study.

Further, the theory of the ecological model posits that within the multiple levels of influence on behavior, the social and the built environment levels operate at multiple levels themselves [25]. Previous research suggested that the influence of built environment attributes on physical activity (e.g., walking and bicycling) should be considered at micro, meso, and macro levels [13]. Thus, based on the ecological 
model of behavior, the built environment characteristics in the nonmotorized travel behavior models developed in this study have been measured utilizing a three-level hierarchy of influence: the micro level (i.e., the neighborhood); the meso level (i.e., the county); and the macro level (i.e., the metropolitan area). The framework of the models also incorporate a three-level hierarchy for the influence of the social environment on nonmotorized travel behavior: the micro level (i.e., the household and the neighborhood); the meso level (i.e., the county); and the macro level (i.e., the metropolitan area). Figures 3 and 4 show the ecological conceptual framework applied to the nonmotorized travel behavior models developed in this study.

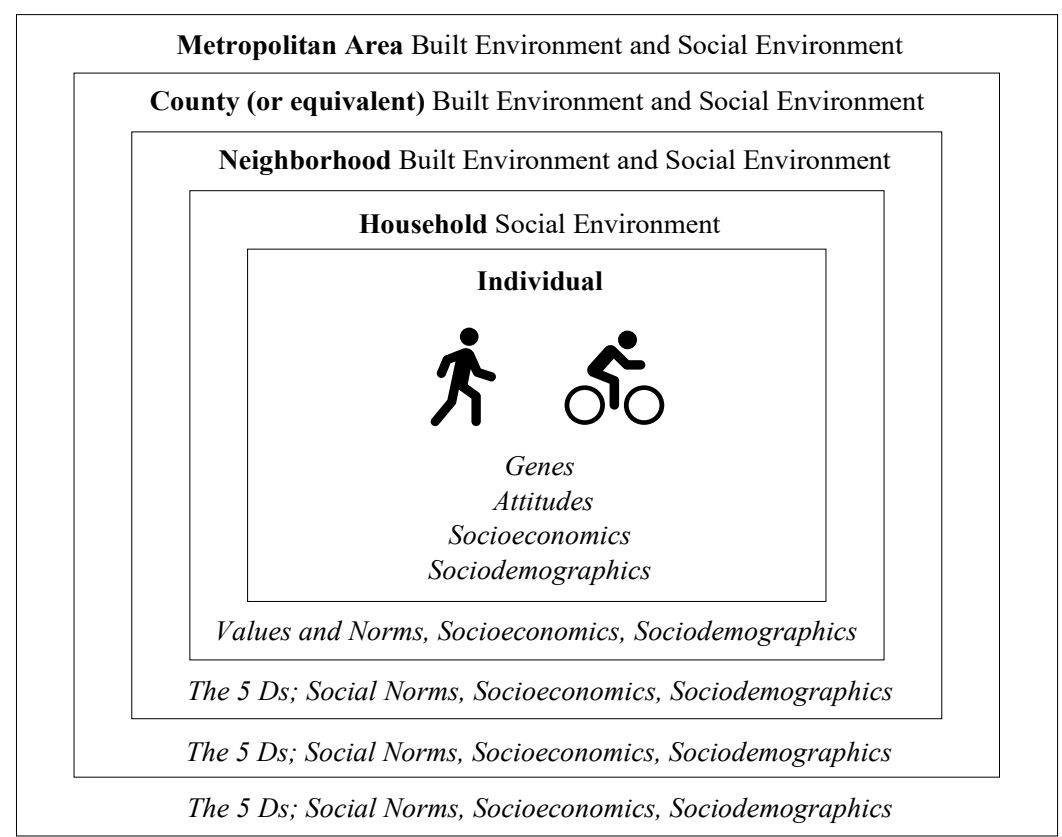

Figure 3. Ecological levels of influence on nonmotorized travel behavior.

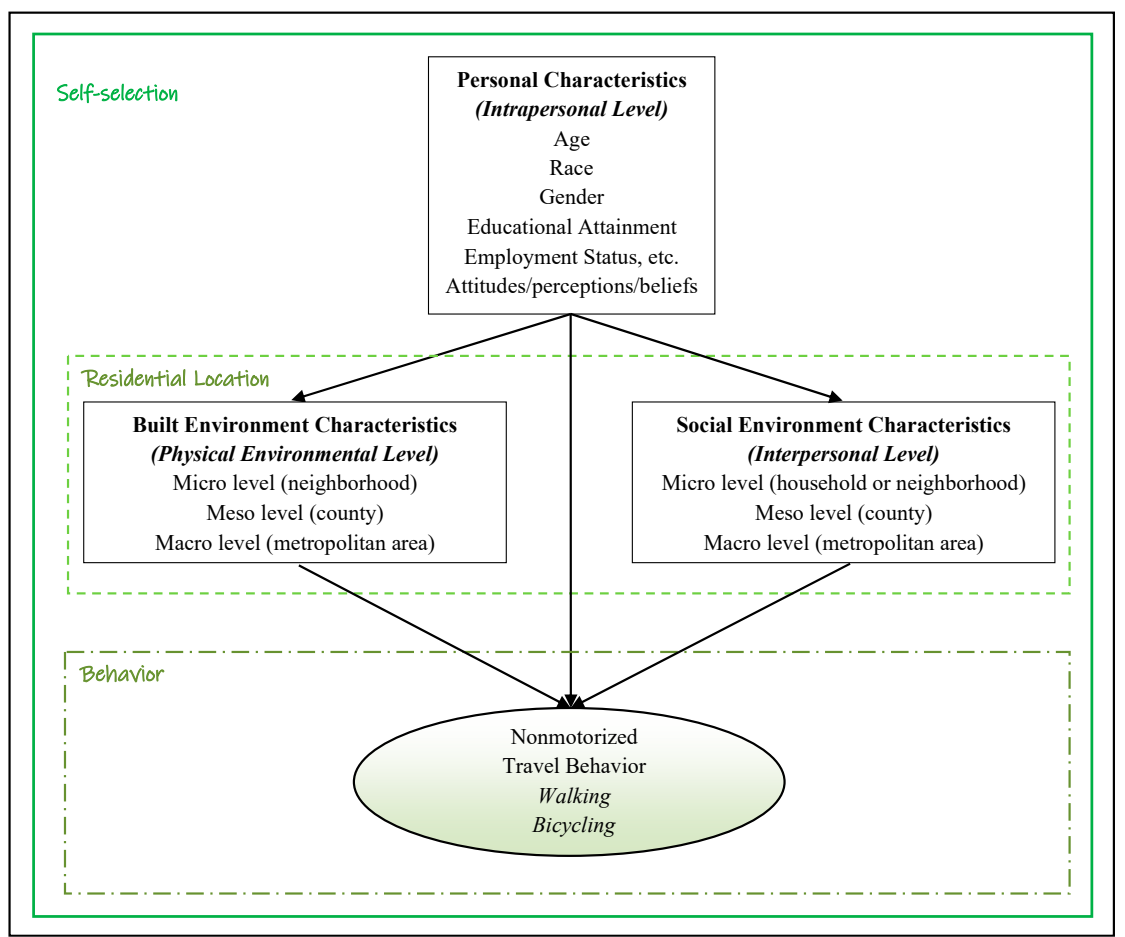

Figure 4. Proposed ecological conceptual framework for levels of influence on nonmotorized travel behavior. 


\subsection{Model Dependent Variables}

Nonmotorized trip mode share for each individual traveler in the Florida 2009 NHTS Add-on sample has been considered for statistical modeling in this study. Separate models for walking and bicycling have been developed based on two dependent variables:

- Individual's daily walking mode share (i.e., number of daily walking trips made by the individual divided by the total number of daily trips made by the individual);

- Individual's daily bicycling mode share (i.e., number of daily bicycling trips made by the individual divided by the total number of daily trips made by the individual).

The 2009 NHTS Add-on data provide information on a total of 24,550 individuals who resided in Florida metropolitan areas. Table 2 lists the frequency of the total number of individuals' daily walking and bicycling trips. The table indicates that the maximum numbers of daily walking and bicycling trips for Florida residents were 18 and 10, respectively. Nonetheless, most individuals did not make any walking or bicycling trips during the travel survey day.

Table 2. Frequency of Florida nonmotorized person trips.

\begin{tabular}{|c|c|c|}
\hline \multirow{2}{*}{ Number of Individual's Trips } & \multicolumn{2}{|c|}{ Frequency } \\
\hline & Walking Trips & Bicycling Trips \\
\hline 0 & 20,369 & 23,972 \\
\hline 1 & 640 & 74 \\
\hline 2 & 2705 & 426 \\
\hline 3 & 155 & 20 \\
\hline 4 & 469 & 39 \\
\hline 5 & 43 & 10 \\
\hline 6 & 107 & 3 \\
\hline 7 & 13 & 2 \\
\hline 8 & 32 & 2 \\
\hline 9 & 2 & 1 \\
\hline 10 & 9 & 1 \\
\hline 11 & 2 & - \\
\hline 12 & 2 & - \\
\hline 13 & - & - \\
\hline 14 & 1 & - \\
\hline 15 & - & - \\
\hline 16 & - & - \\
\hline 17 & - & - \\
\hline 18 & 1 & - \\
\hline Total & 24,550 & 24,550 \\
\hline
\end{tabular}

Notes: - = not applicable; source of data: 2009 NHTS Add-on data.

Further, the 2009 NHTS Add-on data indicate that the average numbers of daily person-level walking and bicycling trips were 0.40 and 0.05 trips, respectively. The average person-level walking mode share for Florida was $8.3 \%$, whereas the average person-level bicycling mode share was $1.1 \%$. These low figures are consistent with the literature that suggests nonmotorized trips occur at very low rates $[17,71]$. Based on the 2009 NHTS Add-on data, Figures 5 and 6 show the average person-level walking and bicycling mode share by Florida county, respectively. 


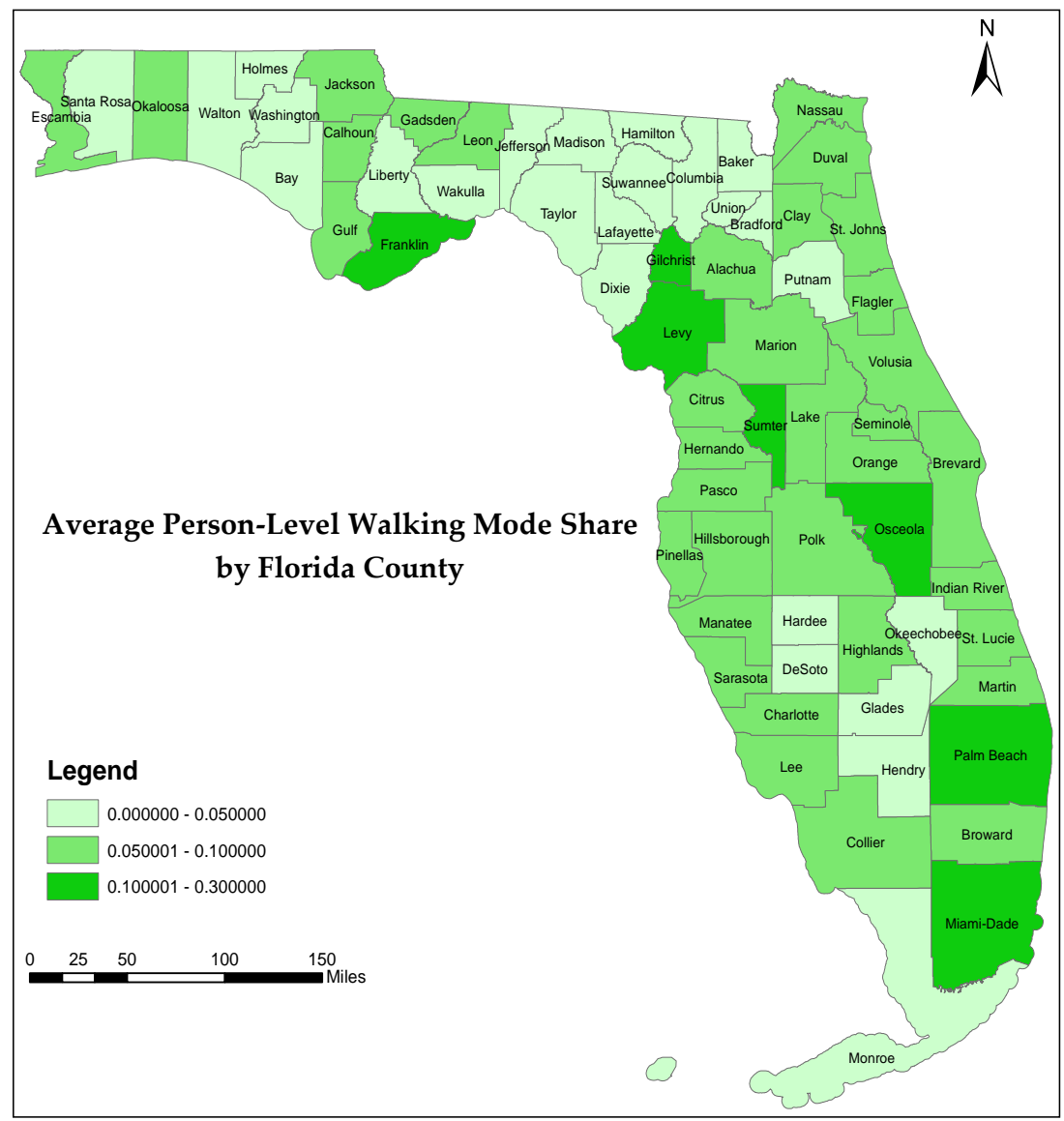

Figure 5. Average person-level walking mode share by Florida county.

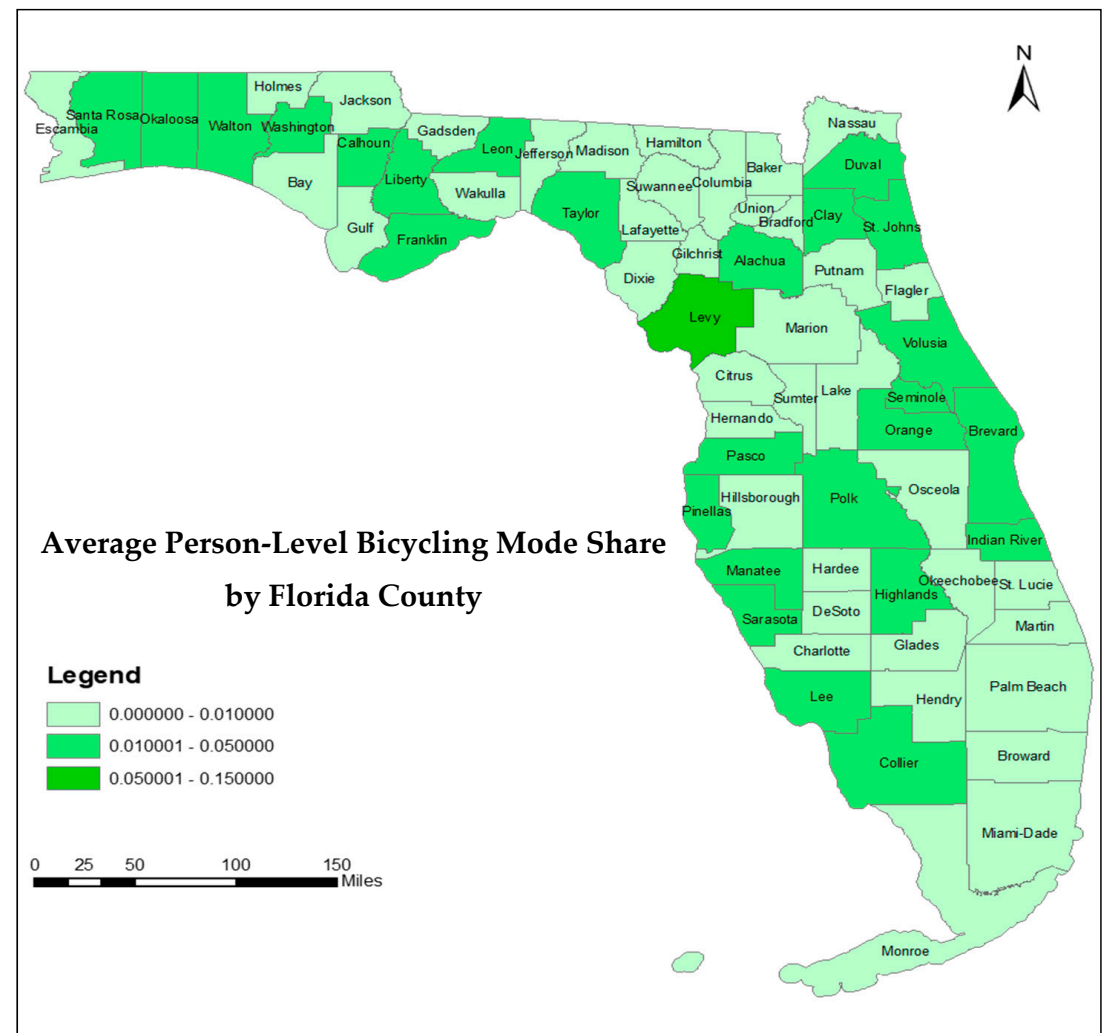

Figure 6. Average person-level bicycling mode share by Florida county. 


\subsection{Model Independent Variables}

As depicted in the model framework (Figures 3 and 4), the independent variables for the models have been chosen based on the principals of the ecological models of behavior, which considers the effects of intrapersonal (i.e., individual) attributes, interpersonal (i.e., social environment) attributes, as well as the spatial environment (i.e., built environment) attributes at hierarchical levels of influence. The independent variables considered for inclusion in the models are discussed below.

\subsubsection{Person-Level Variables (Individual Characteristics)}

To satisfy the ecological model of behavior's emphasis on the intrapersonal level of influence on behavior, person-level characteristics such as biological, sociodemographic and socioeconomic factors, as well as measures representing attitudes and perceptions were considered for inclusion in the models. However, using a complete set of person-level variables that represented all of the above-mentioned characteristics was not possible due to the following data limitations:

- The NHTS Add-on Person File did not include biological data on individual trip-makers; therefore, no variable representing the person's genetic characteristics was included in the models due to unavailability of such data;

- Although the NHTS Add-on Person File provided some data on person's attitudes and perceptions, which can potentially influence one's nonmotorized travel behavior, these data were not usable because a large proportion of observations were missing such information.

Consequently, person-level variables only include sociodemographic and socioeconomic attributes for each individual such as: age, race, gender, employment status, and college education.

\subsubsection{Social Environment Variables}

Micro-level (Household- and Neighborhood-level) Social Environment Variables: The household is considered the most important setting among the social environment levels of influence that determines an individual's behavior [23,72]. Therefore, household control variables have been included in the models to represent the first level of influence (i.e., the micro level) of the social environment on nonmotorized travel behavior. The household-level variables provide information on the household's social environment for each of the Florida 2009 NHTS Add-on respondents including the household's: number of members (i.e., household size), annual income, number of workers, number of vehicles, and number of daily transit trips.

Many past studies highlighted the crucial role of car ownership in nonmotorized travel behavior (see Section 2.1). Thus, variables capturing car ownership levels have been included in the models at influence levels beyond the household. To represent the neighborhood-level social environment, a variable measuring the percentage of zero-vehicle households in the neighborhood has been included in the models.

Meso-level (County-level) Social Environment Variable: To represent the meso-level social environment, a variable has been included in the models that measures the average walking or bicycling density within the county of residence. The inclusion of this variable is intended to operationalize two important behavioral concepts within a nonmotorized travel behavior context. These include: (1) the concept of observational learning-defined within the social cognitive theory [27]; and (2) the concept of contagion perspective-defined by Ross [29]. Representing social norms and sociocultural values, both of these concepts posit that human behavior can be influenced by seeing others perform a certain behavior. Further, past research showed an association between recreational walking and perceptions of others being physically active (e.g., people walking or bicycling) [10].

Therefore, it is hypothesized in the present study that levels of walking and bicycling densities within the county of residence influence an individual's walking and bicycling behavior due to a county-level "cultural" effect. Mitra and Builing [16] who used a measure for walking density in modeling children's nonmotorized trips to school found that children were more likely to walk or 
bicycle in locations where others also walked (i.e., locations with higher walking densities). Thus, it is hypothesized in the present study that individuals who frequently see others walk and bicycle (i.e., individuals who live in counties with higher walking/bicycling densities) engage in such activities at higher rates.

Macro-level (Metropolitan area-level) Social Environment Variables: The macro-level social environment is represented by CBSA-level (i.e., metropolitan area-level) variables including: car ownership levels, income levels, average walking (or bicycling) density, annual public transportation passenger-miles, average gasoline cost, average median age, average percentage of foreign-born population, and average violent crime rate within the CBSA.

The macro-level social environment variables are intended to capture three different features of the social environment within a metropolitan area. These include the metropolitan area's:

1. Sociodemographic and socioeconomic characteristics: together with the CBSA-level car ownership and income variables, variables measuring the CBSA-level average median age and average gasoline cost control for the effects of macro-level sociodemographic and socioeconomic attributes on nonmotorized travel behavior of individuals;

2. Social norms and sociocultural characteristics: as previously mentioned, walking and bicycling density variables have been included in the models as proxies for nonmotorized travel-related social norms and sociocultural values within the metropolitan area and to capture the effects of these concepts on nonmotorized travel behavior of residents. It is hypothesized that in metropolitan-areas where walking/bicycling have higher densities, individuals may be encouraged to walk or bicycle more due to a metropolitan area-level "cultural" effect. The variable measuring the percentage of foreign-born individuals within the CBSA has also been included to control for any cultural differences that may exist among immigrants and the U.S.-born population with respect to nonmotorized travel behavior. This variable has been considered for inclusion in the models based on a study by McMillan [59], who found that having a U.S.-born parent lowered the likelihood of children's nonmotorized travel to school as well as another study by McDonald [57], who found that neighborhoods with a larger immigrant population had higher levels of walking to school among children. Further, the variable measuring the annual public transportation passenger-miles has been included in the analysis to represent the levels of public transit usage within the CBSA - an indicator for a "public transportation culture" within the metropolitan area;

3. Crime-related characteristics: a variable measuring the average CBSA-level violent crime rate has been included in the nonmotorized travel behavior models developed in this study to examine the impact of violent crime levels within the metropolitan area on nonmotorized travel behavior of residents. This variable has been included in the models based on previous research findings suggesting that crime rates within an area may play a role in walking or bicycling of individuals [60].

\subsubsection{Built Environment Variables}

Micro-level (Neighborhood-level) Built Environment Variables: The micro-level built environment variables are based on the SLD data and provide information on the neighborhood-level built environment and land use for each respondent's household location. This study defines the neighborhood as the census block group (CBG) where the household was located. Previous research suggests that the census block group is a relatively small area that approximates a neighborhood [73]. The neighborhood-level built environment variables in the present study include: activity density, entropy, intersection density, pedestrian-friendly network density, local transit service frequency, and distance to local transit (i.e., proximity to transit). These variables represent the $D$ s of the built environment at the neighborhood (i.e., micro) level in the models.

Meso-level (County-level) Built Environment Variables: The meso-level built environment variables are defined based on the county where the household was located. Block group-level built environment and land use measures provided by the SLD were aggregated to obtain the average county-level built 
environment measures for each household location. Aggregation of data at smaller scales to obtain the mean of the explanatory variables at larger scales provides a meaningful contextual variable (i.e., the group mean) for inclusion in multilevel models [74].

The meso-level (i.e., county-level) built environment variables included in the nonmotorized travel behavior models are: average activity density, average entropy, average regional diversity, average intersection density, average pedestrian-friendly network density, average transit service frequency, average local transit accessibility (i.e., average distance to local transit), average automobile accessibility to employment opportunities, average transit accessibility to employment opportunities, and average Walk Score. Together, these variables represent the $D$ s of the built environment at the county (i.e., meso) level.

Macro-level (Metropolitan area-level) Built Environment Variables: Census block group-level built environment measures provided by the SLD were aggregated and averaged to obtain most of the metropolitan area-level (i.e., macro-level) built environment measures for each respondent's household location. This study considers the metropolitan area as the CBSA where the household was located; therefore, the macro-level built environment is represented by CBSA-level variables including: average activity density, average entropy, average total road network density, percentage of small blocks, average automobile accessibility to employment opportunities, average transit accessibility to employment opportunities, average Walk Score, and average roadway congestion index. Together, these variables represent the $D$ s of the built environment at the metropolitan area (i.e., macro) level.

Table 3 provides a list of the variables used in the nonmotorized travel behavior models, along with variable descriptions, computation methods, data sources, and descriptive statistics.

\subsection{Nonmotorized Travel Behavior Models}

\subsubsection{Analytical Methods}

Based on the principles of the ecological model of behavior, this study aims at developing statistical models in such a way as to comprehensively analyze the relationship between individual's nonmotorized travel behavior and measures representing multiple influence levels including the intrapersonal, the interpersonal (i.e., the social environment), and the built environment levels. Previous research suggests that a key element of such relationship is the influence of intrapersonal-level psychological factors such as attitudes and perceptions [37,45,51]. However, as indicated previously, the attitudinal data from the Florida 2009 NHTS Add-on Person File were unusable for the present study due to the large proportions of missing data. The unavailability of attitudinal survey data limits the ability to control for residential self-selection bias in the analysis (i.e., endogeneity bias) as residential self-selection is interwoven with attitudes.

In addition, owing to lack of attitudinal data, the question of correlation or causality of the link between nonmotorized travel behavior and the built environment remains unaddressed. This is because attitudes may simultaneously influence travel choices and residential location choices, which means any correlation observed between nonmotorized travel and the built environment can be the effect of a spurious relationship and not a causal one. Further, the available data are cross-sectional, which limits the ability to test for the causality of the links between nonmotorized travel behavior and built environment factors. 
Table 3. Nonmotorized travel behavior models' variable information.

\begin{tabular}{|c|c|c|c|c|c|}
\hline Variable & Represented Effect & Description/Computation (and Units) & Mean & Std. Dev. & Original Data Source \\
\hline \multicolumn{6}{|c|}{ Individual (i.e., Person) Characteristics } \\
\hline Age & Intrapersonal & Person's age (years) & 46.53 & 21.89 & NHTS \\
\hline Race & Intrapersonal & Person's race: $1=$ white, $0=$ otherwise & 0.76 & 0.43 & NHTS \\
\hline Gender & Intrapersonal & Person's gender: $1=$ male, $0=$ female & 0.46 & 0.50 & NHTS \\
\hline Employment status & Intrapersonal & Employed? $1=$ yes, $0=$ no & 0.61 & 0.49 & NHTS \\
\hline College education & Intrapersonal & College degree? $1=$ yes, $0=$ no & 0.66 & 0.47 & NHTS \\
\hline \multicolumn{6}{|c|}{ Social Environment } \\
\hline \multicolumn{6}{|l|}{ Micro Level: The Household } \\
\hline Number of members & Interpersonal & Count of household members (hh size) & 2.95 & 1.45 & NHTS \\
\hline Number of vehicles & Interpersonal & Vehicles owned by the household & 1.94 & 1.02 & NHTS \\
\hline Number of workers & Interpersonal & Employed persons in the household & 1.27 & 0.91 & NHTS \\
\hline Annual income & Interpersonal & Household annual income category (1,000 s of dollars) & $50-55$ & - & NHTS \\
\hline Number of daily transit trips & Interpersonal & Number of daily transit trips made by all members of the household & 0.11 & 0.61 & NHTS \\
\hline Number of daily nonmotorized trips & Interpersonal & $\begin{array}{l}\begin{array}{c}\text { Number of daily nonmotorized (walking + bicycling) trips made by all members } \\
\text { of the household }\end{array} \\
\end{array}$ & 1.09 & 2.22 & NHTS \\
\hline \multicolumn{6}{|c|}{ Micro Level (Census Block Group): The Neighborhood } \\
\hline Percentage of zero-car hhs & Interpersonal (socioeconomics) & Percentage of households (in CBG) with no private automobiles & 5.67 & 7.90 & SLD \\
\hline \multicolumn{6}{|l|}{ Meso Level (County): The County } \\
\hline Average walking (or bicycling) density & Interpersonal (sociocultural) & $\begin{array}{l}\text { Number of walking (or bicycling) trips in CBG divided by area of CBGs in acre } \\
\text { (averaged for county) }\end{array}$ & $\begin{array}{c}0.0032 \\
(0.0003)\end{array}$ & $\begin{array}{c}0.0025 \\
(0.0001)\end{array}$ & SLD and NHTS \\
\hline \multicolumn{6}{|c|}{ Macro Level (Core Based Statistical Area): The Metropolitan Area } \\
\hline Average percentage of hhs with $2+$ cars & Interpersonal (socioeconomics) & Average percentage of households (in CBSA) with more than two cars & 52.40 & 2.58 & SLD \\
\hline Average percentage of low-wage workers & Interpersonal (socioeconomics) & Average percentage of workers (in CBSA) earning $\leq$ USD 1250/month & 26.32 & 1.86 & SLD \\
\hline Average walking (or bicycling) density & Interpersonal (sociocultural) & $\begin{array}{l}\text { Number of walking (or bicycling) trips in CBG divided by area of CBGs in acre } \\
\text { (averaged for CBSA) }\end{array}$ & $\begin{array}{c}0.0031 \\
(0.0003)\end{array}$ & $\begin{array}{c}0.0021 \\
(0.0001)\end{array}$ & SLD and NHTS \\
\hline Average public transport annual pass-miles & Interpersonal (sociocultural) & Average public transportation annual passenger-miles in CBSA (millions) ${ }^{1}$ & 331.26 & 408.60 & TTI's UMI \\
\hline Average state gasoline cost & Interpersonal (socioeconomics) & Average state gasoline cost (USD/gallon) for CBSA ${ }^{1}$ & 2.80 & 0.05 & TTI's UMI \\
\hline Average median age & Interpersonal (sociodemographics) & Average median age for the entire population in CBSA (years) ${ }^{1}$ & 39.92 & 3.69 & ACS \\
\hline Average percentage of foreign-born & Interpersonal (sociocultural) & Average percentage of population in CBSA that was not born in the U.S. ${ }^{1}$ & 18.54 & 11.98 & ACS \\
\hline Average crime rate & Interpersonal (crime) & Annual number of violent crimes per 100,000 population in CBSA ${ }^{1}$ & 821.67 & 355.51 & UCR and CHR\&R \\
\hline
\end{tabular}


Table 3. Cont

\begin{tabular}{|c|c|c|c|c|c|}
\hline Variable & Represented Effect & Description/Computation (and Units) & Mean & Std. Dev. & Original Data Source \\
\hline \multicolumn{6}{|c|}{ Built Environment } \\
\hline \multicolumn{6}{|c|}{ Micro Level (Census Block Group): The Neighborhood } \\
\hline Activity density & Density & (Employment + housing units) on unprotected land area (acres) & 4.79 & 7.04 & SLD \\
\hline Entropy & Land use diversity & 5-tier employment entropy & 0.59 & 0.31 & SLD \\
\hline Intersection density & Urban design & Auto-oriented intersections $/ \mathrm{mi}^{2}$ & 0.98 & 2.17 & SLD \\
\hline Pedestrian-friendly network density & Urban design & Facility miles of pedestrian-oriented links $/ \mathrm{mi}^{2}$ & 12.89 & 6.71 & SLD \\
\hline Local transit service & Transit accessibility & Aggregate frequency of transit service/mi ${ }^{2}$ & 126.03 & 272.75 & SLD \\
\hline Local transit accessibility & Distance to transit & Distance from centroid to the nearest transit stop (meters) & 662.10 & 162.97 & SLD \\
\hline \multicolumn{6}{|l|}{ Meso Level (County): The County } \\
\hline Average activity density & Density & Average (employment + housing units) on unprotected land (acres) in county & 6.29 & 4.41 & SLD \\
\hline Average entropy & Land use diversity & Average 5-tier employment entropy for county & 0.54 & 0.06 & SLD \\
\hline Average regional diversity & Regional diversity & Average deviation of jobs/population ratio from the regional average & 0.18 & 0.05 & SLD \\
\hline Average intersection density & Urban design & Average automobile-oriented intersections $/ \mathrm{mi}^{2}$ for county & 1.12 & 0.54 & SLD \\
\hline Average pedestrian-friendly network density & Urban design & Average facility miles of pedestrian-oriented links $/ \mathrm{mi}^{2}$ for county & 13.38 & 3.59 & SLD \\
\hline Average transit service & Transit accessibility & Average aggregate frequency of transit service/ $\mathrm{mi}^{2}$ for county & 278.09 & 277.20 & SLD \\
\hline Average local transit accessibility & Distance to transit & Average distance from centroid to the nearest transit stop (meters) & 659.18 & 68.30 & SLD \\
\hline Average temporal auto. accessibility (to jobs) & Destination accessibility & Average number of jobs in county within a 45 -min automobile travel time & $85,896.1$ & $59,076.8$ & SLD \\
\hline $\begin{array}{c}\text { Average temporal transit accessibility (to } \\
\text { jobs) }\end{array}$ & Destination accessibility & Average number of jobs in county within a 45 -min transit commute & $3,750.35$ & $3,327.53$ & SLD \\
\hline Average Walk Score & Destination accessibility & Average Walk Score for county & 10.71 & 17.58 & Walk Score ${ }^{\circledR}$ \\
\hline \multicolumn{6}{|c|}{ Macro Level (Core Based Statistical Area): The Metropolitan Area } \\
\hline Average activity density & Density & Average (employment + housing units) on unprotected land (acres) in CBSA & 6.17 & 3.51 & SLD \\
\hline Average entropy & Land use diversity & Average 5-tier employment entropy for CBSA & 0.54 & 0.05 & SLD \\
\hline Average total road network density & Urban design & Average total road network density for CBSA & 18.83 & 8.39 & SLD \\
\hline Percentage of small blocks & Urban design & Percentage of blocks with an area smaller than $0.01 \mathrm{mi}^{2}$ in CBSA & 58.43 & 8.63 & TIGER/Line \\
\hline Average temporal auto. accessibility (to jobs) & Destination accessibility & Average number of jobs in CBSA within a 45-min automobile travel time & $83,615.8$ & $50,055.7$ & SLD \\
\hline $\begin{array}{l}\begin{array}{c}\text { Average temporal transit accessibility (to } \\
\text { jobs) }\end{array} \\
\text { (t) }\end{array}$ & Destination accessibility & Average number of jobs in CBSA within a 45 -min transit commute & $2,572.9$ & $2,073.9$ & SLD \\
\hline Average Walk Score & Destination accessibility & Average Walk Score for CBSA & 41.52 & 15.06 & Walk Score ${ }^{\circledR}$ \\
\hline Average roadway congestion index & Mobility & Average roadway congestion index for $\mathrm{CBSA}^{1}$ & 1.12 & 0.20 & TTI's UMI \\
\hline
\end{tabular}

Auto. $=$ automobile; $\mathrm{hh}=$ household ${ }^{1}{ }^{\text {multiyear average. }}$ 
Due to the cross-sectional nature of the data and lack of attitudinal data, a sophisticated methodology is needed to more comprehensively examine the link between nonmotorized travel behavior and the built environment and to control for residential self-selection bias in this study. The Structural Equations Modeling (SEM) technique has the capability to simultaneously estimate coefficients for multiple interrelated regression equations and test for the self-selection effect. The SEM methodology has been used in previous travel behavior research to control for self-selection bias and causality in analyses using cross-sectional data and in the absence of personal attitudinal data $[20,43,75]$. Further, with regards to spatial data, the multilevel structure of the models causes a clustered structure for the data. This introduces interdependencies within the data, which can potentially subject the analysis to spatial autocorrelation. Literature suggests that using hierarchical (i.e., multilevel) models can help in statistical treatment of spatial autocorrelation problems, which may exist due to the clustered nature of data [31].

For the above reasons, multilevel Structural Equation Modeling (multilevel SEM) techniques have been employed in nonmotorized travel behavior models developed in this study to explain the complex relationships among person-level walking/bicycling activities and the built as well as the social environment factors, while accounting for interdependency among data from multiple hierarchical levels of influence (i.e., micro, meso, and macro levels). The multilevel SEM approach is a suitable modeling methodology to handle the hierarchical nature of the data as well as the multilevel conceptual model framework used in this analysis. It offers the capability of concurrently analyzing the effect of factors from various levels including those from the individual level and those from the cluster/group level (i.e., contextual effects) on the dependent variable [76].

Employment of the multilevel SEM techniques to examine the link between individuals' nonmotorized travel behavior and built and social environment factors at multiple levels provides many methodological advantages. Most importantly, the application of the multilevel SEM to the comprehensive person-level ecological model framework proposed in this study (Figure 4) allows for controlling for self-selection bias as well as examining the causal links between nonmotorized travel and the built environment at multiple levels of influence. Further, spatial autocorrelation can be accounted for by using the multilevel analysis capabilities embedded in the multilevel SEM.

Past research suggests that the advanced capabilities of the multilevel SEM, which allow for addressing various interdependencies within the model-whether resulting from numerous relationships (i.e., direct and indirect effects) or from a nested data structure (e.g., households nesting within neighborhoods) - make it an appropriate methodology to disentangle the complexity of travel behavior [23]. However, despite having a tremendous potential for application in travel behavior research, this approach has not been taken full advantage of in empirical studies. Very few previous studies have employed multilevel SEM to examine travel behavior [77-79]. The referenced papers suggest that although either SEM or multilevel modeling tools have been applied to travel behavior research, a model combining the SEM and multilevel techniques is rarely found in travel behavior research or even within other aspects of transportation research.

Considering the arguments above and based on the proposed ecological model framework (Figures 3 and 4), the multilevel SEM models developed in this study allow for examination of the causality of the links between nonmotorized travel behavior and the built environment, while simultaneously controlling for self-selection bias. These models relate an individual trip maker's daily walking or bicycling mode share to person-level characteristics as well as built and social environment characteristics at multiple hierarchal levels.

\subsubsection{Model Specification}

Data used in this study are assumed to be clustered as individuals are nested within households and households are nested within similar geographical areas (e.g., neighborhoods, counties, or metropolitan areas). Due to the clustered nature of data, there may be correlations between observations within the same households or observations within the same spatial area (i.e., spatial autocorrelation). These data 
interdependencies are accounted for in this analysis by employing a hierarchical modeling technique (i.e., the multilevel SEM) as well as by consideration of random effects for two clusters: the household and the neighborhood (i.e., census block group).

From a theoretical standpoint, the importance of the consideration of random effects at the household-level in this analysis is further strengthened by the fact that the household is considered the most important social environment setting that determines an individuals' behavior [23,72]. The theoretical basis for consideration of neighborhood random effects comes from the literature providing evidence that neighborhood-level built environment attributes influence nonmotorized travel behavior (see, e.g., [3,16,51]).

With respect to multilevel modeling, this model design introduces three levels: the first level is the individual, the second level is the household, and the third level is the census block group (i.e., neighborhood). The household-level and the neighborhood-level random effects are represented by specifying random intercepts at the household level as well as at the census block group level. Variations in the models can be divided into two components for the household taste: within-household variation and between-household variation, as well as two components for differences in neighborhoods: within-neighborhood variation and between-neighborhood variation. These variations can be captured by the household-level and neighborhood-level random intercepts. Based on Kline [76], this model specification allows for simultaneous analysis of the effect of factors from multiple hierarchical levels including those from the individual level and those from the cluster/group level (i.e., contextual effects) on nonmotorized travel.

With respect to the structural equation modeling, the concepts of dependent and independent variables as defined in an ordinary regression modeling context become blurred. Instead, the SEM specification consists of endogenous and exogenous variables representing logical cause-effect relationships between them. Endogenous variables in an SEM context are defined as variables that need to be explained or predicted, whereas exogenous variables are those that are determined by causes outside of the model and can potentially offer the explanation or prediction desired for endogenous variables in the model $[80,81]$.

A path diagram is often drawn for the SEM, which depicts the structural relationships among variables of interest. Unidirectional arrows represent presumed causal links in the path diagram. The causal links hypothesized in the path diagram should be justified based on theoretical grounds. The path diagram can also include latent variables (i.e., variables that are not observed or measured directly) allowing factor analysis to be embedded in the model.

Figure 7 shows the multilevel SEM model structure (i.e., proposed path diagram) describing the hypothesized causal links among endogenous variables as well as between exogenous and endogenous variables for the nonmotorized travel behavior models developed in this study. Through incorporation of both measurement and structural models, the path diagram presents a latent variable path analysis [80].

According to the conventions of SEM, endogenous and exogenous variables in the path diagram are connected by arrows indicating the direction of influence coming from the exogenous variables and heading toward the endogenous variables. For instance, the direction of an arrow coming from variable $X$ and heading toward variable $Y$ (i.e., $X \rightarrow Y$ ) represents the presumed causal effects (i.e., effect priority) of $\mathrm{X}$ on $\mathrm{Y}$-implying that $\mathrm{X}$ is causally prior to $\mathrm{Y}$ and affects it [76].

Pearson correlation coefficients have been calculated for all original independent variables (i.e., exogenous variables). According to Franke [82], correlation coefficients higher than 0.8 or 0.9 between independent variables are considered as excessively collinear and are indicators of multicollinearity. Therefore, a correlation threshold of $|p|>0.7$ was used to eliminate highly correlated independent variables (i.e., exogenous variables) as suggested by Kim and Susilo [83]. However, two variables that showed a correlation coefficient of 0.84 with each other were retained in the models. These were the variables representing county-level pedestrian-friendly network density (i.e., average facility miles of pedestrian-oriented links $/ \mathrm{mi}^{2}$ ) and county-level activity density 
(i.e., average (employment + housing units) on area of land). The reasons for this decision were that (a) the two variables albeit highly correlated, represent very different aspects of the built environment; and (b) there are theoretical reasons for retaining them since both have been found by previous studies to be key factors in determining nonmotorized travel behavior. Nevertheless, according to Franke [82], one of the capabilities of the SEM technique is that it may deal with collinearity; therefore, it is assumed that the SEM nature of the models developed in this study handles any multicollinearity problems that may exist after elimination of variables based on a correlation threshold of $|p|>0.7$.

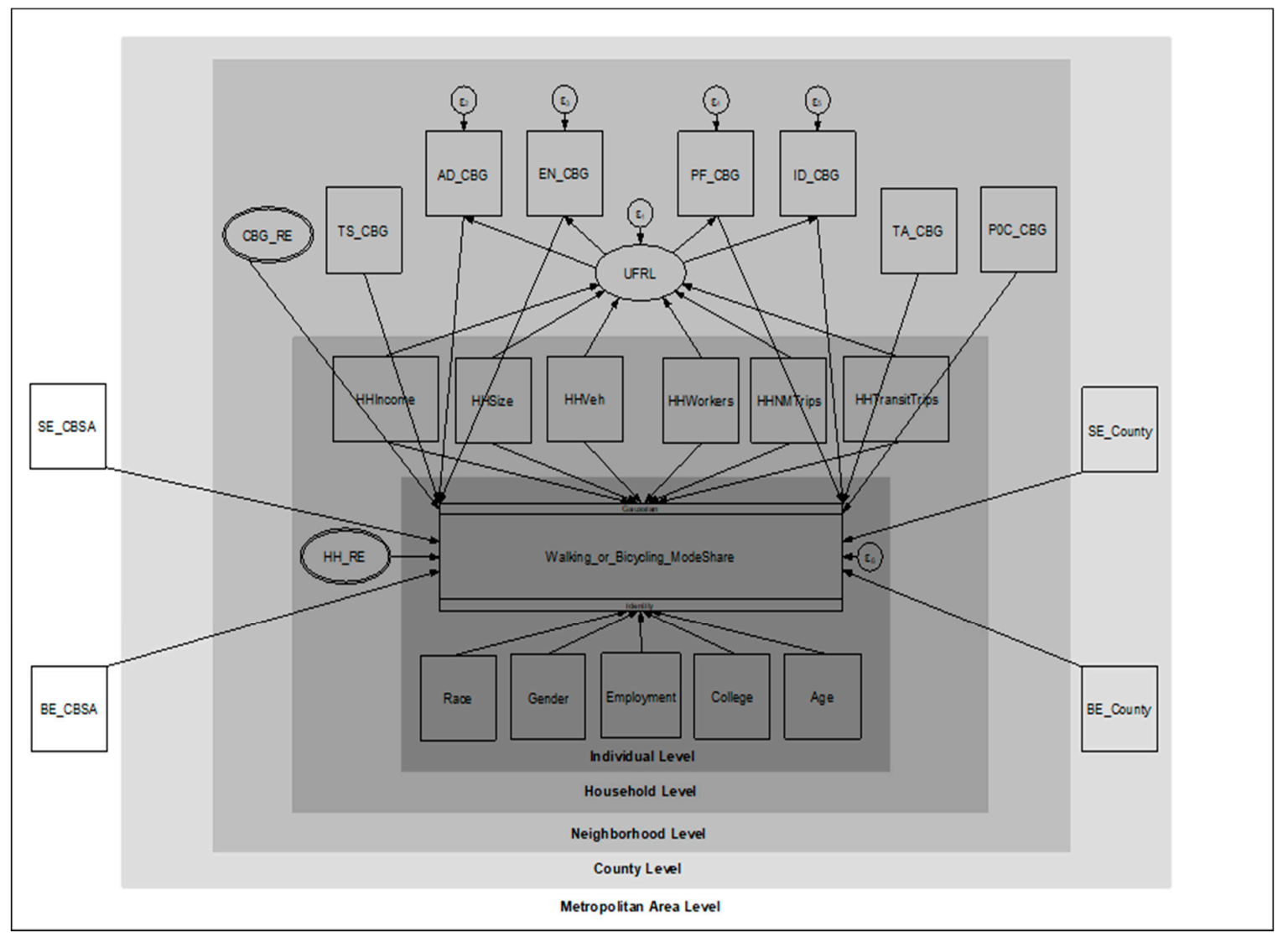

Figure 7. Multilevel Structural Equations Modeling (SEM) structure for nonmotorized travel models. Notes: HH_RE = household-level random effects $\left(\lambda_{\mathrm{HH}}\right) ; \mathrm{CBG} \mathrm{RE}=$ neighborhood-level random effects $\left(\lambda_{\mathrm{CBG}}\right)$; AD_CBG, EN_CBG, PF_CBG, ID_CBG are measurement model variables and represent neighborhood-level activity density, entropy, pedestrian-friendly network density, intersection density, respectively (also, see Equations (1)-(6)); Per conventions of SEM, latent (i.e., unobserved) variables are represented in ovals, whereas measured (i.e., observed) variables are represented in rectangles.

Additionally, any continuous variable with a correctable skewed distribution was normalized by transformation into its naturally logged form before inclusion in the models. Most of the variables however, either showed a normal (or nearly normal distribution) or did not show any improvement in their distribution curve by transformation to naturally logged form (including the dependent variables); therefore, these variables have been included in the models in their original form. Similar to previous studies [84], for independent variables with an original value equal to zero, the zero value was changed to 0.25 before the variable was log-transformed. This is because the natural log of zero is undefined.

The path diagram in Figure 7 can be represented by a simplified regression equation for the person-level daily walking or bicycling mode shares (Equation (1)) and a simplified equation for the latent variable representing the urban form of the residential location as a function of household-level social environment (i.e., household-level socioeconomic factors as well as transit and nonmotorized 
travel behavior factors as measures of household travel culture) (Equation (2)). Equations (1) and (2) constitute the structural model, which is formulated as:

$$
\begin{gathered}
\mathrm{Y}=\beta_{0}+\beta^{\prime}{ }_{1} \mathrm{ED}_{\text {Person }}+\beta^{\prime}{ }_{2} \mathrm{SE}_{\mathrm{HH}}+\beta_{3} \mathrm{SE}_{\mathrm{CBG}}+\beta_{4} \mathrm{SE}_{\mathrm{County}}+\beta^{\prime}{ }_{3} \mathrm{SE}_{\mathrm{CBSA}}+\beta^{\prime}{ }_{4} \mathrm{BE}_{\mathrm{CBG}}+\beta^{\prime}{ }_{5} \mathrm{BE}_{\mathrm{County}}+\beta^{\prime}{ }_{6} \mathrm{BE}_{\mathrm{CBSA}}+\lambda_{\mathrm{HH}}+\lambda_{\mathrm{CBG}}+\varepsilon_{6} \\
\text { Urban Form of Residential Location (UFRL) }{ }_{\text {(Latent Endogenous Variable) }}=\beta^{\prime}{ }_{7} \mathrm{SE}_{\mathrm{HH}}+\varepsilon_{1}
\end{gathered}
$$

where,

$\beta_{0}=$ model intercept;

$\beta^{\prime}{ }_{1}-\beta^{\prime}{ }_{7}=$ column vectors of model path coefficients;

$\beta_{3}$ and $\beta_{4}=$ model parameters for the micro-level (neighborhood-level) and meso-level (county-level) social environment attributes, respectively;

$\lambda_{\mathrm{HH}}$ and $\lambda_{\mathrm{CBG}}=$ household-level and neighborhood-level random effects, respectively;

$\varepsilon_{1}$ and $\varepsilon_{6}=$ model error terms;

$\mathrm{ED}_{\text {Person }}=$ column vector of person-level socioeconomic and sociodemographic attributes;

$\mathrm{SE}_{\mathrm{HH}}, \mathrm{SE}_{\mathrm{CBG}}, \mathrm{SE}_{\text {County }}$, and $\mathrm{SE}_{\mathrm{CBSA}}=$ column vectors of micro-level (household and neighborhood), meso-level (county) and macro-level (metropolitan area) social environment attributes, respectively; $\mathrm{BE}_{\mathrm{CBG}}, \mathrm{BE}_{\mathrm{County}}$, and $\mathrm{BE}_{\mathrm{CBSA}}=$ column vectors of micro-level (neighborhood), meso-level (county-level) and macro-level (metropolitan area) built environment attributes, respectively;

$\mathrm{Y}=$ vector of observed endogenous variable (person-level walking or bicycling mode share).

The Urban Form of Residential Location (UFRL) latent variable is measured by using the main neighborhood-level attributes that represent the overall urban form of the trip-maker's residential neighborhood area. Inclusion of this latent variable in the multilevel SEM nonmotorized travel model allows for estimation of the residential self-selection effect in the absence of attitudinal data.

In construction of the UFRL latent variable, causal links are hypothesized between the household's urban form of residential location and the household-level social environment characteristics (i.e., household taste) including households' socioeconomic and travel culture characteristics. These causal links are included in the model based on the theoretical assumption that household-level social environment factors, such as households' socioeconomic status, influence both travel choices and residential location choices-an assumption supported by previous studies [20,43]. For instance, wealthier individuals who can afford expenses associated with owning private vehicles and long travel distances are more likely to select a low-density suburban neighborhood to reside in, where they can take advantage of cleaner air and spacious land. In contrast, low-income individuals who own no private vehicles may prefer to reside in dense urban neighborhoods conducive to the less costly modes of transportation such as the transit mode [43] or the walking and bicycling modes.

The measurement model for the UFRL latent variable is formulated by Equations (3) through (6) as follows:

$$
\begin{aligned}
& \mathrm{AD}_{\mathrm{CBG}}=\alpha_{1} \mathrm{UFRL}+\varepsilon_{2} \\
& \mathrm{EN}_{\mathrm{CBG}}=\alpha_{2} \mathrm{UFRL}+\varepsilon_{3} \\
& \mathrm{PF}_{\mathrm{CBG}}=\alpha_{3} \mathrm{UFRL}+\varepsilon_{4} \\
& \mathrm{ID}_{\mathrm{CBG}}=\alpha_{4} \mathrm{UFRL}+\varepsilon_{5}
\end{aligned}
$$

where,

UFRL $=$ Urban Form of Residential Location latent variable;

$\mathrm{AD}_{\mathrm{CBG}}, \mathrm{EN}_{\mathrm{CBG}}, \mathrm{PF}_{\mathrm{CBG}}$, and $\mathrm{ID}_{\mathrm{CBG}}=$ neighborhood-level activity density, mixed land use score (entropy), pedestrian-friendly network density, and intersection density, respectively;

$\alpha_{1}-\alpha_{4}=$ measurement model pattern coefficients;

$\varepsilon_{2}-\varepsilon_{2}=$ measurement errors. 
In the measurement model specified above (Equations (3)-(6)), there are four observed built environment characteristics that record the urban form of an individual's residential location and form the latent variable (UFRL). Observed variables used to measure a latent variable are referred to as indicators [76]. The minimum number of indicators to be included in the measurement model for a latent variable (i.e., factor) in an SEM analysis is three [80], with three or four indicators being deemed as a better target [76]. Therefore, the present analysis uses four indicators in the measurement model of the latent variable UFRL.

Equation (2) along with Equations (3)-(6) represent a multiple indicators and multiple independent causes (MIMIC) model where the household-level observed social environment variables (i.e., the $\mathrm{SE}_{\mathrm{HH}}$ variables) determine the UFRL latent variable, and UFRL in turn, determines the observed built environment indicator variables (i.e., built environment characteristics of the residential location neighborhood). The household-level observed social environment variables are treated as predictors of UFRL, and thereby give an estimate of the residential self-selection effect. Through Equations (1)-(6), the model structure allows for the nonmotorized (i.e., walking or bicycling) trip mode shares and the urban form of residential location to be estimated jointly. This means that all components of the path diagram depicted in Figure 7 are simultaneously estimated by the model.

The maximum likelihood estimation (MLE) method—commonly used in practice [85]—is used to develop the multilevel SEMs in the present study. Model estimation is undertaken using the Generalized Structural Equation Modeling (GSEM) mode of the Stata software. In standard linear SEMs, the validity of MLE depends on the SEM meeting the assumption of multivariate (i.e., joint) normality of all model variables, observed and latent. However, the MLE method used in GSEM is applied to a different likelihood function that assumes only conditional normality and does not require the full joint-normality assumption of the standard linear SEMs. The conditional normality assumed in MLE means that latent variables are still assumed to be normally distributed [86].

\section{Results and Discussion}

Table 4 summarizes the estimation results of the multilevel SEMs for the nonmotorized travel behavior models developed in the present study. This section discusses the results of the models in the context of previous findings.

Table 4. Nonmotorized travel behavior models' (multilevel SEMs) estimation results.

\begin{tabular}{|c|c|c|c|c|c|}
\hline \multicolumn{6}{|c|}{ Endogenous Response Variable (Observed): Person-Level Daily Nonmotorized Trip Mode Share } \\
\hline \multirow[b]{2}{*}{ Exogenous and Endogenous Predictor Variables } & \multirow[b]{2}{*}{$\begin{array}{l}\text { Variable Label } \\
\text { (in Figure 7) }\end{array}$} & \multicolumn{2}{|c|}{ Walking Model } & \multicolumn{2}{|c|}{ Bicycling Model } \\
\hline & & $\begin{array}{c}\text { Path } \\
\text { Coefficient }\end{array}$ & $p$-Value & $\begin{array}{c}\text { Path } \\
\text { Coefficient }\end{array}$ & $p$-Value \\
\hline \multicolumn{6}{|c|}{ Individual (i.e., Person) Characteristics } \\
\hline $\begin{array}{c}\text { Age (years) } \\
\text { Person's age (years) }\end{array}$ & Age & $-0.06763^{* * *}$ & 0.000 & $-0.02580^{* * *}$ & 0.000 \\
\hline $\begin{array}{l}\text { Gender }(1=\text { male, } 0=\text { female }) \\
\text { Person's gender: } 1=\text { male, } 0=\text { female }\end{array}$ & Gender & 0.02787 & 0.916 & $0.93991 * * *$ & 0.000 \\
\hline $\begin{array}{l}\text { Employment status }(\text { employed? } 1=\text { yes, } 0=\text { no) } \\
\text { Employed? } 1=\text { yes, } 0=\text { no }\end{array}$ & Employment & $-3.24587^{* * *}$ & 0.000 & $-0.39466^{* * *}$ & 0.013 \\
\hline Number of vehicles & HHveh & $-1.49267^{* * *}$ & 0.000 & $-0.35056^{* * *}$ & 0.000 \\
\hline Annual income & HHIncome & $0.04639 *$ & 0.099 & -0.00747 & 0.561 \\
\hline Number of daily transit trips & HHTransitTrips & $0.93523 * *$ & 0.026 & $0.38484^{* * *}$ & 0.002 \\
\hline \multicolumn{6}{|l|}{ Micro Level (Census Block Group): The Neighborhood } \\
\hline Percentage of zero-car hhs & POC_CBG & 0.05944 * & 0.063 & 0.00562 & 0.556 \\
\hline
\end{tabular}


Table 4. Cont.

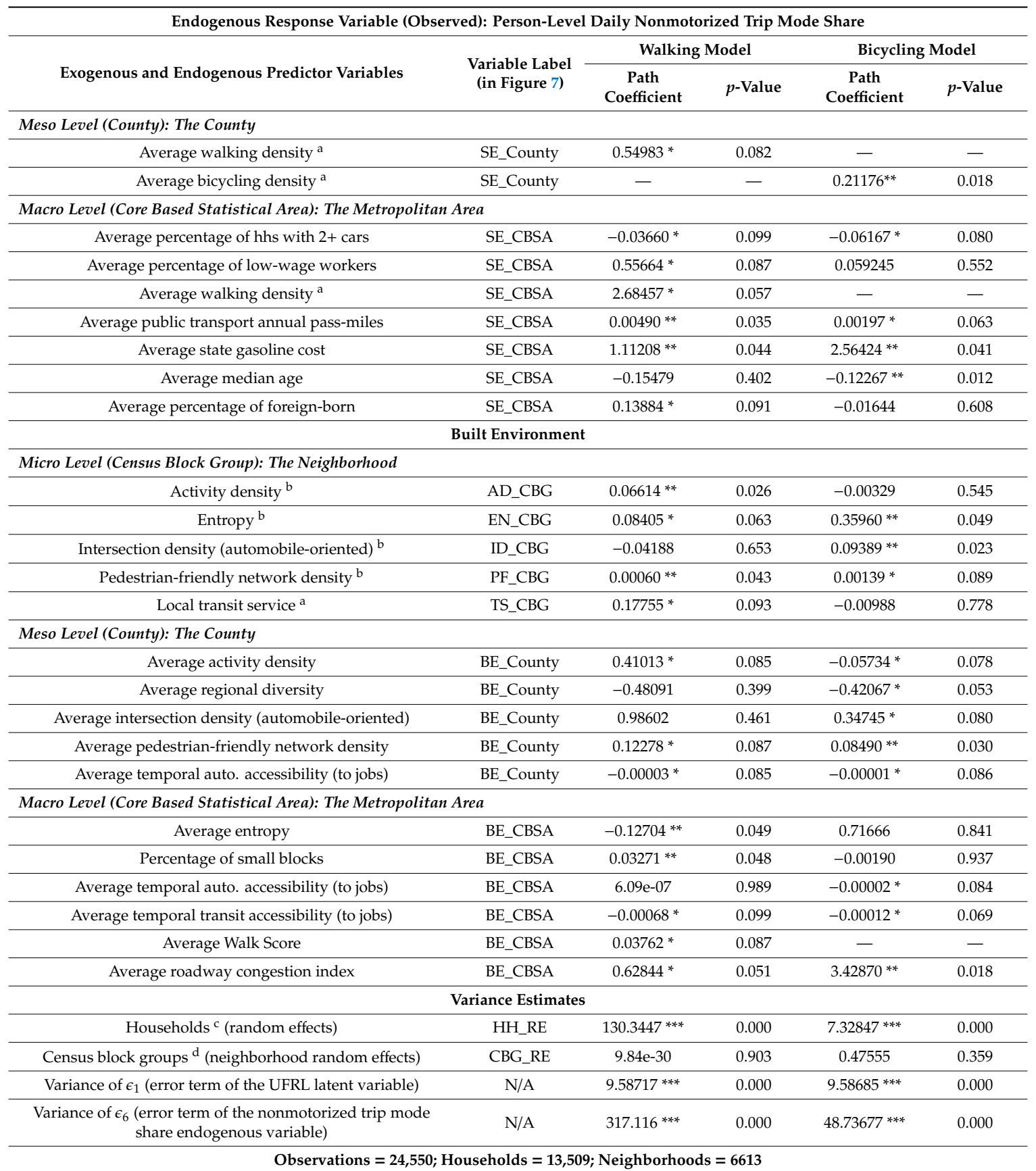

Notes: only variables whose path coefficients were statistically significant in at least one model (i.e., in either walking or bicycling model) are listed in the table; ${ }^{a}$ variable was log-transformed; ${ }^{b}$ endogenous observed variable used in the measurement model of the Urban Form of Residential Location (UFRL) latent variable; ${ }^{c}$ clusters: households;

d clusters: neighborhoods; $-=$ not included in the model; N/A = implied by the figure (but not shown by a label);

$*{ }^{* *},{ }^{* * *}$ path coefficient is significant at the $10 \%, 5 \%$ and $1 \%$ significance level, respectively.

\subsection{Person-Level Variables (Individual Characteristics) Findings}

The results of both the walking and bicycling multilevel SEM models show that as measures of the intrapersonal level of influence on behavior, an individual's sociodemographic and socioeconomic characteristics affect his/her nonmotorized travel behavior. Specifically, individuals' age is negatively linked with their daily nonmotorized mode share. This is a reasonable finding because as an individual gets older, physical activity such as walking and bicycling may get more difficult to perform. Many studies in the past also found age to be negatively correlated with nonmotorized travel [28,29]. 
An individual's race being white is positively linked with his/her daily nonmotorized travel mode share; however, the path coefficient of the race variable does not reach a statistical significance threshold in the bicycling model. This means that race may be a more influential factor in individuals' walking than in their bicycling. With respect to gender, the results indicate that a higher bicycling mode share is linked with being male, as also suggested by many previous studies (see, e.g., [31]). However, gender does not appear to be a statistically significant determinant of individuals' walking mode share. The results also suggest that being employed is linked with lower levels of nonmotorized mode shares (in terms of both walking and bicycling) for individuals. This may be an indication of time limitations that workers face in performing recreational walking and bicycling activities. Further, having a college education has a positive impact on individuals' walking mode share. This is consistent with previous findings that suggested higher education was positively associated with walking $[14,29,70]$. However, the results indicate that the effect of having a college education on an individual's daily bicycling mode share is not statistically significant.

\subsection{Micro-Level (Household/Neighborhood-Level) Social Environment Variables Findings}

The results suggest that household-level socioeconomic characteristics affect individuals' nonmotorized travel behavior. A higher number of private vehicles owned by the household has a negative effect on both walking and bicycling mode shares of individuals. This result corroborates findings by previous studies (see, e.g., [16]). Higher household income levels are positively linked with walking mode share. This result may be an indication of recreational walking by individuals from higher-income households as suggested by previous research [87]. A notable result is the positive direction of influence of the variable representing household's number of daily transit trips on an individual's nonmotorized mode shares (both walking and bicycling). Literature suggests a sizable proportion of all public transit trips involve walking at both ends of the trip, and bicycling can also be a potentially important mode of access to public transit [34]. The results of the nonmotorized mode share models presented here show that as hypothesized, more transit trips encourage more nonmotorized trips. This finding implies that a travel culture within a household that is more oriented toward transit usage leads to higher levels of nonmotorized travel by individuals living within that household.

The car ownership variable at the neighborhood level exhibits a statistically significant path coefficient in the walking mode share model. This variable represents the percentage of households within the neighborhood that own no cars, and its positive sign indicates a positive direction of influence of this variable on individuals' walking mode share. This means that the higher the percentage of zero-car households within the neighborhood, the higher the walking mode share is for residents of that neighborhood. This result also implies that the influence of car ownership has a potential to go beyond the household level.

\subsection{Meso-Level (County-Level) Social Environment Variables Findings}

As measures of the social environment at the county level, the variables representing the average walking and bicycling density within the county show significant effects on individuals' nonmotorized travel behavior. The path coefficients of these variables exhibit a significant positive direction of influence on walking and bicycling mode shares of individuals. As mentioned previously, these variables are intended to operationalize two important behavioral concepts in the models: the concept of observational learning — defined within the social cognitive theory [27]—and the concept of contagion perspective-defined by Ross [29]. Both of these concepts postulate that human behavior can be influenced by seeing others perform a certain behavior. In that sense, the average walking and bicycling density variables are considered proxies for social norms and sociocultural values with respect to nonmotorized travel behavior. Therefore, the results imply that individuals' walking and bicycling are influenced by "cultural effects" within the county of residence. The results also confirm past findings of a positive correlation between individuals' level of nonmotorized travel and higher 
densities of nonmotorized trips within an area [16], as well as a positive association between walking and perceptions of others being physically active (e.g., walking, bicycling, playing sports) [10].

\subsection{Macro-Level (Metropolitan Area-Level) Social Environment Variables Findings}

Based on the model results, many metropolitan area-level social environment variables (i.e., variables representing sociodemographic, socioeconomic, sociocultural, and crime factors) are influential in an individual's nonmotorized travel (i.e., walking and bicycling mode shares). These effects can be discussed in terms of three different features of the social environment within a metropolitan area including the:

1. Sociodemographic and socioeconomic variables effects: the results indicate that as the average median age within the metropolitan area increases, person-level nonmotorized mode share declines. Although the effect is not significant in the walking model, the negative direction of influence is in line with the path coefficient estimate for the Age variable at the individual level (i.e., -0.06763). These results further emphasize the role that age plays in individuals' physical activity such as walking and bicycling. As expected, the metropolitan area-level car ownership variable, which indicates the average percentage of households within the metropolitan area that own more than two cars, is negatively linked with individuals' walking and bicycling mode shares. In addition, the metropolitan area-level income variable (i.e., the Average percentage of low-wage workers variable) exhibits a significant positive effect in the walking mode share model, indicating that lower income levels within the metropolitan area are linked with higher walking mode shares by residents. Moreover, and as hypothesized, the average gasoline price affects nonmotorized travel in a positive direction. This means that as the gasoline price increases within the state where the metropolitan area is located, walking and bicycling mode shares for residents of that metropolitan area also increase. It should be noted that past research found a negative link between increased gasoline price and household-level Vehicle Miles Traveled (VMT) [20]. Thus, the findings of the present study compliment such past empirical findings in suggesting that the cost of gasoline can act as a disincentive in motorized travel, whereas it can be considered an incentive for nonmotorized travel. On the other hand, this finding is also in line with the literature suggesting that the low user-cost of using private vehicle is crucial in discouraging other modes such as walking [28]. The path coefficient of the Average state gasoline cost variable is larger in the bicycling model (2.56424) than in the walking model (1.11208), which implies that higher gasoline prices may lead to generation of more bicycling trips than walking trips. One possible explanation for this can be the effect of trip distance. Longer distances can be traveled by bicycling compared to walking; therefore, individuals may substitute more vehicular trips with bicycle trips if they deem gasoline costs as high. However, further research is needed to examine the effects of gasoline prices on substitution of bicycling trips for vehicular trips considering travel distances;

2. Social norms and sociocultural variables effects: reinforcing the model estimations at the county-level, the path coefficient of the Average walking density variable at the metropolitan area level implies a positive effect of social norms and sociocultural factors on individual's walking. Additionally, a higher percentage of foreign-born population living within the metropolitan area seems to positively influence individuals' walking mode share. Although this finding can be an indication of a lower socioeconomic status of the foreign-born population, it is supported by McMillan [59] who found that the likelihood of children walking and bicycling to school decreased if they had a parent born in the U.S., as well as by McDonald [57] who found that neighborhoods with higher percentages of immigrants had higher levels of walking to school by children. These results provide further support for the influence of cultural norms-in this case, the cultural norms of the country of origin-on nonmotorized travel behavior. The differences between the social norms and travel cultures in the U.S. (which has an automobile-dominated travel culture) and other countries have been discussed in previous studies $[28,56,59]$. These cultural differences seem to exert a statistically significant effect on individuals' walking mode share, as suggested 
by the model estimates in the present analysis. Further, the variable measuring the annual public transportation passenger-miles within a metropolitan area (the Average public transport annual pass-miles variable) has a statistically significant path coefficient in both the walking and bicycling mode share models. The observed positive influence suggests that as transit use within a metropolitan area increases, so does the nonmotorized trip mode share for residents. In the present study, this variable is considered as a proxy for a "public transportation culture" within the metropolitan area. Therefore, this result implies that metropolitan areas with a travel culture that is more oriented toward public transportation promote nonmotorized trips;

3. Crime-related effects: the variable representing the average metropolitan area-level violent crime rate does not reach a statistical significance threshold in the nonmotorized travel models. Previous studies argued that crime rates may play a role in walking or bicycling [60]. However, the results of the present case study suggest that any impact that crime levels within the metropolitan area may have on nonmotorized travel behavior diminishes after the effects of other metropolitan area-level factors (e.g., socioeconomic, sociodemographic, and sociocultural factors) are controlled for. This result is in line with empirical findings by Nehme et al. [10] who found that walking and the number of violent crimes within the neighborhood were not significantly associated.

Cumulatively, the findings presented in Sections 4.2-4.4 lend credibility to the argument that the social environment at multiple levels of influence (i.e., micro, meso, and macro levels) plays a key role in nonmotorized travel behavior.

\subsection{Micro-Level (Neighborhood-Level) Built Environment Variables Findings}

Consistent with findings of many past studies (see, e.g., $[3,16,51]$ ), the results of the models developed in the present study suggest that neighborhood-level built environment attributes impact nonmotorized travel behavior. Particularly, the results indicate that living in neighborhoods with higher activity (i.e., residential and employment) densities, higher extent of land use mix (entropy), higher densities of pedestrian-friendly street networks, and higher frequencies of local transit service is linked with higher walking mode shares for residents. In addition, living in neighborhoods with a higher extent of land use mix, higher levels of street connectivity (higher intersection density), and denser pedestrian-oriented network designs is linked with higher bicycling mode shares for residents. As an example of coefficient interpretation, the path coefficient of the neighborhood-level Pedestrian-friendly network density variable in the bicycling model (0.00139) means that as the value of "facility miles of pedestrian-oriented links $/ \mathrm{mi}^{2}$ " within the neighborhood increases by one unit, the person-level daily bicycling mode share (a continuous number between 0 and 100, which represents the mode share as a percentage) increases by approximately 0.0014 percentage points.

\subsection{Meso-Level (County-Level) Built Environment Variables Findings}

The models' estimations suggest that built environment characteristics at the county level also influence nonmotorized travel. Specifically, higher activity densities and denser pedestrian-oriented networks at the county level are related to higher person-level walking mode shares, whereas higher levels of accessibility to employment by means of automobiles are related to lower walking mode shares. On the other hand, higher county-level activity density negatively affects individuals' bicycling mode share. This result lends support to the hypothesis that built environment characteristics can impact walking and bicycling travel behavior in different directions.

Further, higher county-level regional diversity as well as higher levels of accessibility to employment by means of automobiles within the county lead to lower bicycling mode shares for individuals, whereas higher pedestrian friendliness and increased connectivity of the county street network promote the individual-level bicycling mode share. 


\subsection{Macro-Level (Metropolitan Area-Level) Built Environment Variables Findings}

The results of the nonmotorized travel behavior models suggest that higher mixed-use development within the entire metropolitan area is related to lower walking mode shares for residents. The negative direction of influence can be capturing a substitution effect; residents may be driving to additional and more remote destination options (provided by the higher extent of mixed land use within the metropolitan area) instead of walking to nearby local destinations.

As hypothesized, higher Walk Scores and higher percentages of small blocks within the metropolitan area affect individuals' walking mode share in a positive direction. These results indicate that increased walking mode shares for residents are related to higher walkability, increased destination accessibility by means of walking, and better street connectivity within the metropolitan area.

Moreover, higher transit accessibility to jobs within the entire metropolitan area leads to lower walking and bicycling mode shares for residents. The negative direction of influence can be capturing the effect of driving to transit stations rather than walking or bicycling to stations. The results further indicate that higher automobile accessibility to jobs within the metropolitan area discourages bicycling, which is an expected result.

The path coefficient of the Average total road network density variable is statistically insignificant in both the walking and bicycling models, which is in line with findings of previous studies suggesting that density of major roads did not have an impact on walking/bicycling travel $[16,43]$.

The positive sign of the path coefficients of the Average roadway congestion index in the models is expected. In the present study, this variable represents mobility within the metropolitan areas. Literature suggests that the speed (i.e., extent of mobility) of alternative modes influences travel behavior such as modal choice [28]. Therefore, it was hypothesized in this study that increased roadway congestion levels (which indicate lower levels of speed and mobility) lead to increased levels of nonmotorized mode shares. Model estimations confirm that hypothesis and indicate that increased congestion (i.e., decreased mobility) within the metropolitan area positively affects walking and bicycling mode shares of residents. Past empirical research found that increased congestion within the metropolitan area led to lower household-level VMT [20]. This lends support to the finding of the present study that increased levels of congestion (i.e., lower levels of mobility) within a metropolitan area promote nonmotorized travel—perhaps through discouraging vehicular trips.

\subsection{Random Effects}

As seen from Table 4, the variances of the household-level random intercepts are estimated by the walking and bicycling mode share models to be 130.3447 and 7.32847 , respectively. Despite being less than the corresponding estimated error variances (317.116 in the walking model and 48.73677 in the bicycling model), the estimated variances are sufficiently large to not be disregarded.

These results indicate that after controlling for the various variables in the models, there remains some household-level variance in the models that is unaccounted for. Thus, there appears to be significant variation in averages of the person-level walking/bicycling mode shares across households. These results indicate that household-level random effects (i.e., random differences across households) play a significant role in nonmotorized travel behavior of individuals.

However, the variances of the neighborhood-level random intercepts are small and statistically insignificant. Therefore, it can be inferred that after controlling for the variables in the models, neighborhood-level random effects (i.e., random differences between neighborhoods) do not play a crucial role in nonmotorized travel behavior of individuals. This finding stands in contrast to the findings of previous research, which suggested that random differences between neighborhoods played a small but statistically significant role in nonmotorized travel behavior-at least in terms of walking trips [88].

Therefore, further research is probably needed to clarify the role of neighborhood random effects in nonmotorized travel behavior. Nonetheless, the present findings emphasize the key role that the 
household—as the first and the most influential social environment setting—plays in nonmotorized behavior of individuals.

\subsection{The Self Selection Effect}

As the model path diagram (Figure 7) shows, the latent variable UFRL was created to represent the overall urban form of the residential location of the individual trip-makers. This latent variable is measured from four observed land use and built environment variables at the neighborhood level through Equations (3)-(6).

It is hypothesized that the UFRL latent variable is influenced by households' social environment characteristics (i.e., $\mathrm{SE}_{\mathrm{HH}}$ variables). Together, Equation (2) along with Equations (3)-(6), represent the multiple indicators and multiple independent causes (MIMIC) model specified to examine the self-selection effect in the nonmotorized travel behavior models.

Table 5 presents estimation results of the measurement model for the UFRL latent variable (i.e., results of Equations (3)-(6)) as well as those of the related structural model, which is assumed to quantify the self-selection effect (Equation (2)).

Table 5. Self-selection effects (MIMIC model results).

\begin{tabular}{|c|c|c|c|}
\hline To Variable & From Variable & $\begin{array}{c}\text { Pattern } \\
\text { Coefficient }\end{array}$ & $p$-Value \\
\hline \multicolumn{4}{|c|}{ Measurement Model: Latent Variable-Urban Form Residential Location (UFRL) } \\
\hline $\mathrm{AD}_{\mathrm{CBG}}$ (activity density) $^{\mathrm{a}}$ & UFRL & 1 (constrained $^{\mathrm{b}}$ & - \\
\hline $\mathrm{EN}_{\mathrm{CBG}}(\text { entropy score })^{\mathrm{a}}$ & UFRL & $0.01895^{* * *}$ & 0.000 \\
\hline $\mathrm{PF}_{\mathrm{CBG}}$ (ped.-friendly network density) ${ }^{\mathrm{a}}$ & UFRL & $1.47382 * * *$ & 0.000 \\
\hline $\mathrm{ID}_{\mathrm{CBG}}$ (intersection density) ${ }^{\mathrm{a}}$ & UFRL & $0.07395^{* * *}$ & 0.000 \\
\hline \multicolumn{4}{|c|}{ Structural Model: Self-Selection Effect } \\
\hline UFRL & Number of members (household size) & -0.03342 & 0.475 \\
\hline UFRL & Number of household vehicles & $-0.70283^{* * *}$ & 0.000 \\
\hline UFRL & Number of household workers & $0.32297^{* * *}$ & 0.000 \\
\hline UFRL & Annual income of the household & $-0.02504^{* * *}$ & 0.008 \\
\hline UFRL & Number of hh daily transit trips & $0.64754^{* *}$ & 0.011 \\
\hline UFRL & Number of hh daily nonmotorized trips & $0.08994^{* * *}$ & 0.001 \\
\hline
\end{tabular}

Notes: ${ }^{a}$ neighborhood-level built environment variable; ${ }^{\mathrm{b}}$ by SEM conventions, the pattern coefficient of the activity density variable in the UFRL measurement model is constrained to be equal to $1 ; * * * * *$ path coefficient is significant at the $5 \%$ and $1 \%$ significance level, respectively; also, owing to utilization of the same data and same variables, the estimation results of the measurement model for the UFRL latent variable and the structural model that captures the self-selection effect are almost identical in the walking and bicycling mode share models.

In measurement models with a MIMIC factor (i.e., latent variable), some observed indicators are specified as effects of the latent variable, whereas other indicators are specified as causes of the latent variable [89]. Accordingly, the neighborhood-level built environment variables in the measurement model (i.e., the $\mathrm{AD}_{\mathrm{CBG}}, \mathrm{EN}_{\mathrm{CBG}}, \mathrm{PF}_{\mathrm{CBG}}$, $\mathrm{ID}_{\mathrm{CBG}}$ variables) were specified as the effects of the UFRL latent variable, whereas the household-level social environment variables (i.e., number of members, number of workers, vehicle ownership, annual income, level of transit usage, and level of nonmotorized travel) were specified as the cause of UFRL. Thus, the results of the model are interpreted in such way to reflect these cause-effect considerations.

The direction of the hypothesized causal effects from household social environment variables to the UFRL latent variable (which is itself measured from the built environment variables) represents the residential self-selection behavior (see [43]). The results of the self-selection effect analysis (i.e., the structural portion of the MIMIC model presented in Table 5 show that household social environment characteristics - often termed households' taste in transportation research—influence households' residential location choice. Most notably, the exogenous variables representing household's number of daily transit and nonmotorized trips exhibit positive effects on the UFRL latent variable. 
The positive direction of these effects implies that households with more transit and more nonmotorized trips choose to live in more walkable and bikeable urban neighborhoods, which often also foster transit use. In other words, the results confirm that households' travel culture impacts their residential location choice. Thus, despite not being able to make direct inferences about the influence of individuals' travel attitudes and preferences on their residential locations (due to unavailability of attitudinal survey data in this study), it can be inferred from the model estimations that households with a travel culture more oriented toward nonmotorized and transit trips self-select into residential locations that facilitate their travel culture (which most likely represents their travel attitudes and preferences).

In addition, the exogenous household-level socioeconomic variables show statistically significant effects on households' residential location choice. The variables representing the number of vehicles owned by the household and the household's annual income have negative effects on the UFRL latent variable, suggesting that households with more private vehicles and higher annual income levels choose to reside in suburban neighborhoods where they can enjoy cleaner air, ample parking space, larger homes, as well as less congested and less noisy streets. These results confirm an argument by Wang [43] who suggested that higher-income individuals may be willing to live in low-density suburban neighborhoods. In contrast, the variable representing the number of household workers exerts a positive effect on UFRL latent variable. This implies that households with more workers tend to locate closer to dense urban neighborhoods where higher levels of mixed-use development provide better access to more destinations including employment centers.

With respect to the measurement model estimations, the variables from which the UFRL latent variable is measured show statistically significant pattern coefficients with positive signs. This means that urban neighborhoods are neighborhoods that are more compact with higher levels of mixed-use development, increased density of pedestrian-friendly network, and better street connectivity. These results are in line with how literature characterizes an urban neighborhood, particularly with the definition of "urban neighborhood" as provided by Wang [43]. The referenced study considered an urban neighborhood a neighborhood with high density, high mixed land use, high intersection density (i.e., better street connectivity) as opposed to a low-density, residential-only, suburban neighborhood with not much street connectivity [43].

The direction of the pattern coefficients in the measurement model of UFRL from the neighborhood built environment variables and the direction of effects of household characteristics including car ownership, number of workers, and number of transit trips on residential location choice (i.e., UFRL variable) corroborate findings in previous research [20]. This consistency in findings lends a degree of confidence to the results obtained in the present study.

Together, the estimation results of the structural model and the measurement model presented in Table 5 support the hypothesis that both the neighborhood built environment and residential self-selection influence nonmotorized travel behavior-a finding that corroborates past research findings [42,53-55].

\section{Conclusions}

The main hypothesis of this study was that nonmotorized travel behavior has become dependent on environmental factors from larger-scale spatial areas. More specifically, the main goal of this study was twofold: (1) to examine the role of larger-scale spatial areas (i.e., county-level and metropolitan area-level) built and social environment factors in nonmotorized travel behavior in addition to that of neighborhood-level built and social environment factors; and (2) to investigate the role of self-selection in nonmotorized travel behavior as well as the causal links between nonmotorized travel and the built environment within a comprehensive model framework that incorporates factors from various spatial levels.

The principles of the ecological model of behavior-a variant of the social cognitive theory [27]—were employed to develop the conceptual framework for this study. As an advanced statistical tool, the multilevel SEM method was utilized to estimate models capturing the link between 
nonmotorized travel behavior of residents of several metropolitan areas in Florida and built as well as social environment factors at multiple levels (i.e., micro, meso, and macro levels) of influence. The study conclusions are discussed next in terms of research findings and policy implications as well as research contributions and limitations.

\subsection{Research Findings and Contributions}

The study findings suggest that individuals' sociodemographic and socioeconomic characteristics (i.e., the intrapersonal level of ecological influence) affect their nonmotorized travel behavior. The results provide evidence that individuals' walking and/or bicycling mode shares are linked with their age, race, gender, employment status, and college education.

Further, the research findings also indicate that contextual effects-such as those of the social environment (i.e., the interpersonal level of ecological influence) at multiple levels-play a role in people's nonmotorized travel behavior.

Representing the micro-level social environment, households' socioeconomic factors such as vehicle ownership and annual income influence individuals' nonmotorized trips. Vehicle ownership levels within the neighborhood also have an impact, particularly on walking trips. Further, the findings provide support for concluding that the impact of vehicle ownership and income go beyond the household and neighborhood boundaries as macro-level (i.e., metropolitan area-level) measures of these factors are also influential in individuals' nonmotorized trips. Other macro-level sociodemographic and socioeconomic attributes such as the median age and the gasoline price within the metropolitan area also impact individuals' nonmotorized trip mode share.

As representatives of the social environment, sociocultural factors such as the travel culture within the household, county, or metropolitan area of residence also prove influential in individuals' walking and bicycling mode shares. At the micro level, the extent of household's daily transit trips is linked with individuals' nonmotorized trips mode share. This is perhaps capturing the positive influence of the "transit travel culture" within a household on walking/bicycling trips of household members. Further, the densities of nonmotorized trips within the county (i.e., the meso level) and the metropolitan area (i.e., the macro level) are linked with individuals' nonmotorized trips. Moreover, the annual public transportation passenger-miles—considered in this study as a proxy for a "public transportation travel culture" within the metropolitan area-is also influential in nonmotorized trip mode shares of individuals. The percentage of foreign-born population living within the metropolitan area (i.e., macro level) also affects individuals' walking, which can be capturing the influence of the cultural norms of the country of origin on people's nonmotorized travel behavior. Therefore, it can be concluded that nonmotorized travel behavior is influenced by "sociocultural effects", which include social norms concerning nonmotorized travel and public transit that exist within the household as well as within the area of residence at multiple spatial levels.

The findings also provide evidence of the impact of household-level random effects in individuals' nonmotorized trips, emphasizing the importance of households' tastes in nonmotorized travel behavior. This conclusion is supported by the literature suggesting that the most important setting within the individual's social environment is the household [1,23,44,72].

Together, these findings accentuate the importance of examining the relationship between nonmotorized travel behavior and environmental factors within an ecological model framework, which allows the assessment of the role of multiple levels of the social environment on the observed behavior (e.g., nonmotorized travel behavior).

With respect to the role of the built environment in nonmotorized travel behavior, the study findings also highlight the advantages of using an ecological model framework, which incorporates multiple levels of the built environment. Considering the estimation results of the multilevel SEM models, it can be concluded that individuals' nonmotorized travel behavior is linked with the built environment attributes (i.e., the built environment level of ecological influence) of their place of residence at multiple spatial levels. 
Particularly, the findings imply that within the sample analyzed in this study, causal links exist between individuals' nonmotorized trip mode share and the micro-level (i.e., neighborhood-level) built environment attributes including the extent of: compactness, mixed-use development, pedestrian friendliness and connectivity of the street network, and access to public transit service within the neighborhood. Further, the findings reveal that individuals' nonmotorized travel behavior is influenced not just by the character of their neighborhood but also by the spatial structure and the context of the county or metropolitan area which their neighborhood is a part of. More specifically, the study findings suggest that individuals' nonmotorized trip mode share is linked with a few meso-level (i.e., county-level) built environment characteristics including the extent of: compactness, regional diversity, connectivity and pedestrian friendliness of the street network, and automobile accessibility to employment opportunities within the county. The macro-level built environment characteristics with potential causal relations with individuals' nonmotorized trips include the extent of: mixed-use development, connectivity of the street network, pedestrian friendliness and walkability of the streets, roadway congestion, and automobile as well as transit accessibility to employment opportunities within the metropolitan area. With regards to the role of mixed-use development in nonmotorized trips, it can be concluded from the model results that the stimulating effect of mixed-use development on individuals' nonmotorized trips, which exists at lower spatial scales such as the neighborhood, diminishes at higher spatial scales such as the metropolitan area. One explanation for this can be that more land use diversity within metropolitan areas may encourage residents to make more vehicular trips to various, more remote destinations rather than using nonmotorized modes to visit local destinations. Therefore, an optimal threshold may exist for cities to become mixed in terms of land use to promote nonmotorized travel.

Overall, these findings support the hypotheses of this study that in addition to the built environment of the neighborhood (i.e., micro level), the built environment of the county (i.e., meso level) and the metropolitan area (i.e., the macro level) also play a role in shaping individuals' nonmotorized travel behavior. Thus, the research findings provide evidence that in analyzing the link between the built environment and walking or bicycling trips, going beyond the neighborhood boundaries may be essential. Considering the hierarchical nature of the levels of influence (i.e., the micro, meso, and macro levels), the importance of using an ecological model framework in examining the link between nonmotorized travel and the built environment is, therefore, re-emphasized through these findings. In addition, the findings suggest that the link between the residential location's built environment and nonmotorized travel behavior may be a causal one. In other words, it can be concluded from research findings that the effects of the built environment on nonmotorized travel go beyond the neighborhood boundaries (as they are significant at higher spatial levels), and the links between the two go beyond mere correlations (as they imply causality-at least in this sample).

Additionally, the findings suggest that residential self-selection plays a decisive role in nonmotorized travel behavior. Based on the model results, self-selection of individuals is shaped by household social environment attributes (i.e., household's taste). Further, the findings indicate that individuals' self-selection influences their choice of residential location. More specifically, the findings suggest that households with a travel culture more oriented toward nonmotorized and transit trips self-select into neighborhoods that facilitate their travel culture-which probably represents their attitudes and preferences toward travel. Based on these findings, it can be inferred that both the built environment and self-selection influence nonmotorized travel behavior, as suggested by several past studies [42,53-55].

These findings provide support for the hypotheses of this study that self-selection can play a key role in nonmotorized travel behavior and the link between nonmotorized travel behavior and the built environment may be a causal one. This is not to imply that the findings of the present study guarantee causal relations but rather to emphasize the potential of existence of such relations between nonmotorized travel behavior and the built environment factors. In drawing conclusions with regards to causality and self-selection, one should be mindful of two caveats in this analysis: 
(1) the use of cross-sectional data (which limits the ability to make causal inferences); and (2) the unavailability of attitudinal survey data (which limits the ability to make inferences about self-selection). Nevertheless, it is assumed that the capabilities offered by the multilevel SEM techniques combined with the comprehensive ecological framework of the models allowed for adequate examination of the causal links between nonmotorized travel behavior and built environment factors as well as for addressing the self-selection bias in this analysis. Thus, it is concluded that the successful estimation of the multilevel SEMs in the present study have yielded policy-relevant findings.

This research contributes to the body of knowledge on the role of the built environment in nonmotorized travel by deriving a comprehensive theoretical framework from the principles of the ecological model of behavior to systematically examine the link between walking and bicycling mode shares and built environment factors at various spatial levels. The main contribution of this research is looking at the bigger picture-the position of the neighborhood with respect to the region and the metropolitan area in which it is located-when analyzing nonmotorized trips. Such a research framework advances the body of knowledge on nonmotorized travel behavior by assisting policymakers in determining the role of the overall context and structure of urban areas in walking and bicycling activities of residents. The implications of these findings can provide insights for those who are tasked with making and executing decisions related to improving the equity and affordability of the transportation opportunities within communities. This can assist policy decision-makers as well as urban/transportation planning and engineering professionals who aim at enhancing sustainability and livability of their communities to more appropriately and more efficiently allocate available resources toward that goal.

\subsection{Policy Implications}

Analyses presented in this paper provide a useful and systematic approach for researchers and policy analysts to determine the most effective interventions for promoting walking and bicycling trips. The ecological models developed in this study highlight the role of the built environment-at various spatial levels-in nonmotorized travel behavior. As a key strength of the ecological model of behavior, this focus on multiple hierarchical levels of influence broadens options for interventions [25]. To infer policy conclusions and develop suitable interventions, the effect of each level of influence can be considered separately or in combination with the effects of other levels as estimated in the analysis.

As regards the built environment, findings from the present study provide compelling evidence that modifications to the Ds of the built environment-at multiple spatial levels-can influence nonmotorized travel behavior. These findings imply that policies concentrating on interventions that target the built environment within the neighborhood (i.e., micro level), the county (i.e., meso level), and the metropolitan area (i.e., macro level) to make them more supportive of nonmotorized and transit trips can promote walking and/or bicycling by residents.

According to the study results, at the neighborhood (i.e., micro) level, such interventions include changes to the neighborhood built environment that: increase compactness (i.e., density), increase mixed-use development (i.e., land use diversity), increase walkability and bikeability (i.e., destination accessibility), increase pedestrian friendliness of the street network (i.e., design), increase connectivity of the street network (i.e., design), and increase access to transit facilities and services (e.g., shorter distance to transit stations, more frequency of service).

At the county (i.e., meso) level, key interventions to promote walking and/or bicycling include changes to the built environment within the county that: increase compactness (i.e., density) in areas with existing low residential and employment densities, increase pedestrian friendliness of the street network (i.e., design), and improve connectivity of the street network (i.e., design).

Further, a salient finding of this study is that the built environment characteristics of the metropolitan area (i.e., macro level) influence nonmotorized travel behavior of residents. Therefore, these characteristics should also be considered in decisions regarding policy and intervention development. In this respect, findings suggest that walking and bicycling can be promoted through 
interventions to modify the built environment within the entire metropolitan area in such ways that: improve connectivity of the street network (i.e., design), increase walkability (i.e., destination accessibility), and facilitate accessibility to transit by means of nonmotorized modes, particularly by walking (e.g., shorter distance to transit stations).

According to the study findings, the social environment characteristics within the residential area are also influential in promoting nonmotorized trips. More specifically, the findings provide evidence that individuals' walking and bicycling trips are influenced by factors representing the socioeconomic status and sociocultural values-particularly with respect to travel culture-within the county and/or metropolitan area. Thus, interventions targeting these characteristics can also be considered in policy decisions. Based on the study findings, stimulating walking and bicycling trips through changes to the social environment within the area of residence include interventions that: encourage more use of nonmotorized modes of travel through observational learning (i.e., seeing others engage in walking and bicycling activities), encourage more use of public transportation modes, discourage automobile ownership, and discourage the use of the automobile mode through increasing gasoline costs.

Overall, evidence found in this study supports the idea that nonmotorized trips can be promoted through interventions that target the environment-built and social. The findings imply that a built environment inimical to nonmotorized modes of travel can discourage walking and bicycling trips, whereas an amenable one can foster these trips. To promote nonmotorized modes of travel, therefore, policies and interventions that can make the built environment more supportive of walking and bicycling activities should be pursued, while policies encouraging sprawling urban developments and automobile-oriented urban designs should be avoided.

In terms of the social environment, the study findings imply that communities with travel cultures more oriented toward nonmotorized and public transportation modes foster walking and bicycling trips. Thus, policies and programs that incentivize non-automobile mobility and disincentivize automobile dependency within the society can be developed. This includes policies that can potentially increase the cost of automobile ownership and use. An argument by Pucher et al. [28] is well-suited here: the low cost of using automobiles in the U.S. (e.g., low gasoline costs) is a key factor in discouraging other modes of travel including the nonmotorized ones, whereas higher costs of automobile use in Europe is a factor that makes car ownership less essential and increases the tendency to use nonmotorized modes [28].

The analysis framework and findings of the present study can assist policy decision-makers aiming to increase levels of walking and bicycling within their communities in assessment of interventions that involve changes to the built and/or social environment. Yet, the main point of the study findings must be re-emphasized: more effective policies to promote walking and/or bicycling seem to be the ones that consider the overall form of the metropolitan area in addition to that of the county and the neighborhood of residence. Put differently, to promote nonmotorized trips, interventions to modify the built environment should be considered not just within the neighborhood but also in combination with those within the county as well as throughout the entire metropolitan area. In addition, interventions targeting the built environment should be considered in combination with those targeting the social environment and not in isolation. This provides a seamless and integrated policy framework, which can help urban/transportation planning decision-makers to find more potent intervention strategies and to create urban environments that are more amenable to walking and bicycling. Policies developed based on such a comprehensive framework can yield the most efficient use of resources and the most optimized solutions.

\subsection{Study Limitations and Future Research}

Analyses presented in this study, albeit significant contributions to the research on the link between nonmotorized travel behavior and the built environment, have a few limitations.

Main data-related limitations include the use of cross-sectional data and lack of usable data on attitudes and perceptions. First, cross-sectional data were used in the analyses, which provide 
a snapshot of the information at a single point in time. By nature, cross-sectional analyses can capture correlations but do not allow for a full examination of causal relationships. The employment of SEM techniques-to a degree-allowed for examination of causal links between nonmotorized travel behavior and built environment factors in the present analysis. One should be mindful, however, that although causality is implied by the results obtained from samples analyzed for this research, "proof of causality" for broader, real world processes is not provided by findings of this study. This is-according to Kline [76] - due to the impracticality of any single study to meet all the conditions required for inference of causality. Therefore, future research can benefit from utilization of longitudinal data, which allow for a more intensive investigation of causal relationships and can provide more reliable evidence of the causal links between nonmotorized travel behavior and the built environment. Second, owing to lack of attitudinal data, the effect of residential self-selection may have not been thoroughly captured in this study. Absent attitudinal data, a sophisticated model framework and advanced statistical methods were used in an attempt to capture self-selection bias. This included application of the latent variable approach within an SEM model structure and defining the self-selection effect as a function of households' social environment (i.e., households' tastes), which can influence residential location choices. The SEM tools have been used in past travel behavior research to account for self-selection bias in analysis of cross-sectional data and in the absence of attitudinal data $[20,43,75]$. Nevertheless, as residential self-selection is intertwined with attitudes, using attitudinal survey data can undoubtedly improve the analysis, and allow future research to fully control for any potential self-selection bias.

Further, due to data limitations and the scope of this research, several other built (and social) environment factors that can potentially impact walking and bicycling trips were not included in the models. Among such factors are: extent of toll roads and amount of toll fees as well as parking availability and fees (which can affect nonmotorized trips in CBD areas). While including an entire universe of variables is not feasible in any modeling analysis, consideration of built and social environment variables that were not included in the present study may help find other key influential variables and ensure that a more parsimonious model is developed.

In addition, although this study controlled for the influence of built and social environments on nonmotorized travel behavior, it did not address an equally important aspect of the environment, which can potentially impact walking and bicycling - the natural environment. Weather and climate, topography, and extent of vegetation-all may influence the decision to walk or bicycle and the levels of such activities. Future research can extend the scope of the study by including such potentially contributing factors to gain a deeper and more comprehensive understanding of the impact of the environment as a whole on nonmotorized travel behavior.

Moreover, the data used for developing the nonmotorized travel behavior models in this study came only from a few metropolitan areas in Florida and may not be fully representative of the U.S. metropolitan areas. Thus, it may be best to apply some caution with respect to the potential transferability of the results to other cities. Data from additional metropolitan areas and other states within the U.S. can be analyzed in the future to investigate the link between nonmotorized travel behavior and various levels (i.e., micro, meso, macro levels) of the built and social environment within an ecological model framework. An analysis based on such enhanced data, which offer more variation-particularly with regards to macro-level built and social environment factors, can provide insights into the generalizability of the findings of the present study.

Lastly, although the core concept of the ecological model includes a policy level as a key level of influence on behavior (see [25]), due to lack of data on policy measures, a policy level was not included in the ecological nonmotorized travel behavior models developed in this study. Future research can examine the effects of policy (e.g., parking, housing, bicycling policies) on nonmotorized travel behavior within an integrated model framework with multiple levels of influence such as the one proposed and tested in the present study. 
Author Contributions: Conceptualization, J.M. and L.Z.; methodology, J.M.; software, J.M.; validation, J.M., and L.Z.; formal analysis, J.M.; investigation, J.M.; resources, J.M. and L.Z.; data curation, J.M.; writing-original draft preparation, J.M.; writing - review and editing, J.M. and L.Z.; visualization, J.M.; supervision, L.Z. All authors have read and agreed to the published version of the manuscript.

Funding: This research received no external funding.

Acknowledgments: The authors thank the anonymous reviewers for their insightful comments and valuable suggestions, which helped improve the quality of this work.

Conflicts of Interest: The authors declare no conflict of interest.

\section{References}

1. National Research Council (U.S.); Committee on Physical Activity, Land Use and Institute of Medicine (U.S.). Does the Built Environment Influence Physical Activity? Examining the Evidence (No. 282); Transportation Research Board: Washington, DC, USA, 2005.

2. Chen, C.; Gong, H.; Paaswell, R. Role of the built environment on mode choice decisions: Additional evidence on the impact of density. Transportation 2008, 35, 285-299. [CrossRef]

3. Schneider, R.J. Local environment characteristics associated with walking and taking transit to shopping districts. J. Transp. Land Use. 2015, 8, 125-147. [CrossRef]

4. National Household Travel Survey, Explore Person Trips (PT) Statistics. Available online: https://nhts.ornl. gov/person-trips (accessed on 15 August 2020).

5. Milne, A.M.M.; Melin, M. Bicycling \& Walking in the United States 2016 Benchmark Report. 2016. Available online: https://bikeleague.org/sites/default/files/2016BenchmarkingReport_web.pdf (accessed on 15 August 2020).

6. U.S. Department of Transportation, Bureau of Transportation Statistics. Transportation Statistics Annual Report 2016. Available online: https://www.bts.gov/sites/bts.dot.gov/files/docs/TSAR_2016.pdf (accessed on 15 August 2020).

7. Environmental Protection Agency, Inventory of US Greenhouse Gas Emissions and Sinks: 1990-2015. Available online: https://www.epa.gov/ghgemissions/inventory-us-greenhouse-gas-emissions-and-sinks1990-2015 (accessed on 15 August 2020).

8. Andersen, L.B.; Schnohr, P.; Schroll, M.; Hein, H.O. All-cause mortality associated with physical activity during leisure time, work, sports, and cycling to work. Arch. Intern. Med. 2000, 160, 1621-1628. [CrossRef] [PubMed]

9. Lindström, M. Means of transportation to work and overweight and obesity: A population-based study in southern Sweden. Prev. Med. 2008, 46, 22-28. [CrossRef]

10. Nehme, E.K.; Oluyomi, A.O.; Calise, T.V.; Kohl III, H.W. Environmental correlates of recreational walking in the neighborhood. Am. J. Health Promot. 2016, 30, 139-148. [CrossRef]

11. Liao, Y.; Tsai, H.H.; Wang, H.S.; Lin, C.P.; Wu, M.C.; Chen, J.F. Travel mode, transportation-related physical activity, and risk of overweight in Taiwanese adults. J. Transp. Health 2016, 3, 220-225. [CrossRef]

12. Tajalli, M.; Hajbabaie, A. On the relationships between commuting mode choice and public health. J. Transp. Health 2017, 4, 267-277. [CrossRef]

13. King, A.C.; Stokols, D.; Talen, E.; Brassington, G.S.; Killingsworth, R. Theoretical approaches to the promotion of physical activity: Forging a transdisciplinary paradigm. Am. J. Prev. Med. 2002, 23, 15-25. [CrossRef]

14. Ewing, R.; Schmid, T.; Killingsworth, R.; Zlot, A.; Raudenbush, S. Relationship between urban sprawl and physical activity, obesity, and morbidity. Am. J. Health Promot. 2003, 18, 47-57. [CrossRef]

15. Greenwald, M.; Boarnet, M. Built environment as determinant of walking behavior: Analyzing nonwork pedestrian travel in Portland, Oregon. Transp. Res. Rec. J. Transp. Res. Board 2001, 1780, 33-41. [CrossRef]

16. Mitra, R.; Buliung, R.N. Built environment correlates of active school transportation: Neighborhood and the modifiable areal unit problem. J. Transp. Geogr. 2012, 20, 51-61. [CrossRef]

17. Friedman, B.; Gordon, S.P.; Peers, J.B. Effect of neotraditional neighborhood design on travel characteristics. Transp. Res. Rec. J. Transp. Res. Board 1994, 1466, 63-70.

18. Cervero, R.; Gorham, R. Commuting in transit versus automobile neighborhoods. J. Am. Plan. Assoc. 1995, 61, 210-225. [CrossRef]

19. Krizek, K.J. Operationalizing neighborhood accessibility for land use-travel behavior research and regional modeling. J. Plan. Educ. Res. 2003, 22, 270-287. [CrossRef] 
20. Nasri, A.; Zhang, L. Assessing the impact of metropolitan-level, county-level, and local-level built environment on travel behavior: Evidence from 19 US urban areas. J. Urban. Plan. Dev. 2014, 141, 04014031-1-04014031-10. [CrossRef]

21. Cao, X.; Mokhtarian, P.L.; Handy, S.L. Examining the impacts of residential self-selection on travel behaviour: A focus on empirical findings. Transp. Rev. 2009, 29, 359-395. [CrossRef]

22. Ewing, R.; Meakins, G.; Hamidi, S.; Nelson, A.C. Relationship between urban sprawl and physical activity, obesity, and morbidity-update and refinement. Health Place 2014, 26, 118-126. [CrossRef] [PubMed]

23. Van Acker, V.; Van Wee, B.; Witlox, F. When transport geography meets social psychology: Toward a conceptual model of travel behaviour. Transp. Rev. 2010, 30, 219-240. [CrossRef]

24. Guo, J.Y.; Bhat, C.R. Operationalizing the concept of neighborhood: Application to residential location choice analysis. J. Transp. Geogr. 2007, 15, 31-45. [CrossRef]

25. Sallis, J.F.; Owen, N.; Fisher, E.B. Ecological models of health behavior. In Health Behavior and Health Education: Theory, Research, and Practice, 4th ed.; Glanz, K., Rimer, B., Viswanath, K., Eds.; Jossey-Bass, A Wiley Imprint: San Francisco, CA, USA, 2008; pp. 465-485.

26. Ajzen, I. The theory of planned behavior. Organ. Behav. Hum. Decis. Process. 1991, 50, 179-211. [CrossRef]

27. Bandura, A. Social Foundations of Thought and Action: A Social Cognitive Theory; Prentice-Hall: Englewood Cliffs, NJ, USA, 1986.

28. Pucher, J.; Komanoff, C.; Schimek, P. Bicycling renaissance in North America? recent trends and alternative policies to promote bicycling. Transp. Res. Part. A Policy Pract. 1999, 33, 625-654. [CrossRef]

29. Ross, C.E. Walking, exercising, and smoking: Does neighborhood matter? Soc. Sci. Med. 2000, 51, $265-274$. [CrossRef]

30. Cervero, R.; Duncan, M. Walking, bicycling, and urban landscapes: Evidence from the San Francisco Bay Area. Am. J. Public Health 2003, 93, 1478-1483. [CrossRef] [PubMed]

31. Moudon, A.V.; Lee, C.; Cheadle, A.D.; Collier, C.W.; Johnson, D.; Schmid, T.L.; Weather, R.D. Cycling and the built environment, a US perspective. Transp. Res. Part. D Transp. Environ. 2005, 10, 245-261. [CrossRef]

32. McDonald, N.C.; Brown, A.L.; Marchetti, L.M.; Pedroso, M.S. US school travel, 2009: An assessment of trends. Am. J. Prev. Med. 2011, 41, 146-151. [CrossRef]

33. Scuderi, M.G. Bayesian Approaches to Learning from Data How to Untangle the Travel Behavior and Land Use Relationships. Ph.D. Thesis, University of Maryland, College Park, MD, USA, 2005.

34. Pucher, J.; Buehler, R.; Merom, D.; Bauman, A. Walking and cycling in the United States, 2001-2009: Evidence from the National Household Travel Surveys. Am. J. Public Health 2011, 101, S310-S317. [CrossRef]

35. Biehl, A.; Ermagun, A.; Stathopoulos, A. Modelling determinants of walking and cycling adoption: A stage-of-change perspective. Transp. Res. Part. F Traffic Psychol. Behav. 2018, 58, 452-470. [CrossRef]

36. Dill, J.; Carr, T. Bicycle commuting and facilities in major US cities: If you build them, commuters will use them. Transp. Res. Rec. J. Transp. Res. Board 2003, 1828, 116-123. [CrossRef]

37. Cao, X.; Mokhtarian, P.; Handy, S. Neighborhood design and the accessibility of the elderly: An empirical analysis in Northern California. Int. J. Sustain. Transp. 2010, 4, 347-371. [CrossRef]

38. Ewing, R.; Cervero, R. Travel and the built environment: A meta-analysis. J. Am. Plan. Assoc. 2010, 76, 265-294. [CrossRef]

39. Badoe, D.; Miller, E. Transportation-land-use interaction: Empirical findings in North America, and their implications for modeling. Transp. Res. Part. D Transp. Environ. 2000, 5, 235-263. [CrossRef]

40. Boer, R.; Zheng, Y.; Overton, A.; Ridgeway, G.K.; Cohen, D.A. Neighborhood design and walking trips in ten US metropolitan areas. Am. J. Prev. Med. 2007, 32, 298-304. [CrossRef] [PubMed]

41. Weinberger, R.; Sweet, M. Integrating walkability into planning practice. Transp. Res. Rec. J. Transp. Res. Board 2012, 2322, 20-30. [CrossRef]

42. Cao, X.; Handy, S.L.; Mokhtarian, P.L. The influences of the built environment and residential self-selection on pedestrian behavior: Evidence from Austin, TX. Transportation 2006, 33, 1-20. [CrossRef]

43. Wang, K. Causality between built environment and travel behavior: Structural equations model applied to Southern California. Transp. Res. Rec. J. Transp. Res. Board 2013, 2397, 80-88. [CrossRef]

44. Handy, S.L. Critical assessment of the literature on the relationships among transportation, land use, and physical activity. Transportation Research Board and the Institute of Medicine Committee on Physical Activity, Health, Transportation, and Land Use. Resour. Pap. TRB Spec. Rep. 2005, 282, 1-81. 
45. Handy, S.L.; Xing, Y. Factors correlated with bicycle commuting: A study in six small US cities. Int. J. Sustain. Transp. 2011, 5, 91-110. [CrossRef]

46. Pritchard, R.; Frøyen, Y. Location, location, relocation: How the relocation of offices from suburbs to the inner city impacts commuting on foot and by bike. Eur. Transp. Res. Rev. 2019, 11, 14. [CrossRef]

47. Cervero, R. Walk-and-ride: Factors influencing pedestrian access to transit. J. Public Transp. 2001, 3, 1. [CrossRef]

48. Ryan, S.; Frank, L.F. Pedestrian environments and transit ridership. J. Public Transp. 2009, 12, 3. [CrossRef]

49. Durand, C.P.; Tang, X.; Gabriel, K.P.; Sener, I.N.; Oluyomi, A.O.; Knell, G.; Porter, A.K.; Hoelscher, D.M.; Kohl, H.W. The association of trip distance with walking to reach public transit: Data from the California household travel survey. J. Transp. Health 2016, 3, 154-160. [CrossRef] [PubMed]

50. Plantinga, A.J.; Bernell, S. The Association between urban sprawl and obesity: Is it a two-way street? J. Reg. Sci. 2007, 47, 857-879. [CrossRef]

51. Lund, H. Testing the claims of new urbanism: Local access, pedestrian travel, and neighboring behaviors. J. Am. Plan. Assoc. 2003, 69, 414-429. [CrossRef]

52. Handy, S.; Cao, X.; Mokhtarian, P. Correlation or causality between the built environment and travel behavior? Evidence from Northern California. Transp. Res. Part. D Transp. Environ. 2005, 10, 427-444. [CrossRef]

53. Cao, X. Exploring causal effects of neighborhood type on walking behavior using stratification on the propensity score. Environ. Plan. A 2010, 42, 487-504. [CrossRef]

54. Fan, Y. The Built Environment, Activity Space, and Time Allocation: An Activity-based Framework for Modeling the Land Use and Travel Connection. Ph.D. Thesis, The University Of North Carolina, Chapel Hill, NC, USA, 2007.

55. Chatman, D.G. Residential choice, the built environment, and nonwork travel: Evidence using new data and methods. Environ. Plan. A 2009, 41, 1072-1089. [CrossRef]

56. McMillan, T.E. Urban form and a child's trip to school: The current literature and a framework for future research. J. Plan. Lit. 2005, 19, 440-456. [CrossRef]

57. McDonald, N.C. Children's Travel: Patterns and Influences. Ph.D. Thesis, University of California, Berkeley, CA, USA, 2005.

58. McAlister, A.L.; Perry, C.L.; Parcel, G.S. How individuals, environments, and health behaviors interact: Social cognitive theory. In Health Behavior and Health Education: Theory, Research, and Practice, 4th ed.; Glanz, K., Rimer, B., Viswanath, K., Eds.; Jossey-Bass, A Wiley Imprint: San Francisco, CA, USA, 2008; pp. 169-188.

59. McMillan, T.E. Walking and Urban Form: Modeling and Testing Parental Decisions about Children's Travel. Ph.D. Thesis, University of California, Irvine, CA, USA, 2003.

60. Joh, K.; Boarnet, M.G.; Nguyen, M.T. Interactions between Race/Ethnicity, Attitude, and Crime: Analyzing Walking Trips in the South Bay Area. In Proceedings of the 88th Annual Meeting of the Transportation Research Board, Washington, DC, USA, 11-15 January 2009.

61. Pikora, T.; Giles-Corti, B.; Bull, F.; Jamrozik, K.; Donovan, R. Developing a framework for assessment of the environmental determinants of walking and cycling. Soc. Sci. Med. 2003, 56, 1693-1703. [CrossRef]

62. Götschi, T.; de Nazelle, A.; Brand, C.; Gerike, R.; Consortium, P. Towards a comprehensive conceptual framework of active travel behavior: A review and synthesis of published frameworks. Curr. Environ. Health Rep. 2017, 4, 286-295.

63. Wang, X.; Conway, T.L.; Cain, K.L.; Frank, L.D.; Saelens, B.E.; Geremia, C.; Kerr, J.; Glanz, K.; Carlson, J.A.; Sallis, J.F. Interactions of Psychosocial Factors with Built Environments in Explaining Adolescents' Active Transportation; Preventive Medicine: Amsterdam, The Netherland, 2017; Volume 100, pp. 76-83. [CrossRef]

64. Mitra, R.; Nash, S. Can the built environment explain gender gap in cycling? An exploration of university students' travel behavior in Toronto, Canada. Int. J. Sustain. Transp. 2019, 13, 138-147. [CrossRef]

65. U.S. Census Bureau, Core-Based Statistical Areas. Available online: https://www.census.gov/topics/housing/ housing-patterns/about/core-based-statistical-areas.html (accessed on 15 August 2020).

66. Carr, L.J.; Dunsiger, S.I.; Marcus, B.H. Walk score ${ }^{\mathrm{TM}}$ as a global estimate of neighborhood walkability. Am. J. Prev. Med. 2010, 39, 460-463. [CrossRef] [PubMed]

67. Carr, L.J.; Dunsiger, S.I.; Marcus, B.H. Validation of Walk Score for estimating access to walkable amenities. Br. J. Sports Med. 2011, 45, 1144-1148. [CrossRef]

68. Duncan, D.T.; Aldstadt, J.; Whalen, J.; Melly, S.J.; Gortmaker, S.L. Validation of Walk Score ${ }^{\circledR}$ for estimating neighborhood walkability: An analysis of four US metropolitan areas. Int. J. Environ. Res. Public Health 2011, 8, 4160-4179. [CrossRef] [PubMed] 
69. Manaugh, K.; El-Geneidy, A. Validating walkability indices: How do different households respond to the walkability of their neighborhood? Transp. Res. Part. D Transp. Environ. 2011, 16, 309-315. [CrossRef]

70. Wasfi, R.A.; Dasgupta, K.; Eluru, N.; Ross, N.A. Exposure to walkable neighbourhoods in urban areas increases utilitarian walking: Longitudinal study of Canadians. J. Transp. Health 2016, 3, 440-447. [CrossRef]

71. Kuzmyak, J.R.; Walters, J.; Bradley, M.; Kockelman, K.M. Estimating Bicycling and Walking for Planning and Project Development: A Guidebook (Project 08-78); NCHRP: Washington, DC, USA, 2014.

72. Gochman, D.S. Handbook of Health Behavior Research 1: Personal and Social Determinants; Plenum Press: New York, NY, USA, 1997.

73. Zick, C.D.; Hanson, H.; Fan, J.X.; Smith, K.R.; Kowaleski-Jones, L.; Brown, B.B.; Yamada, I. Re-visiting the relationship between neighbourhood environment and BMI: An instrumental variables approach to correcting for residential selection bias. Int. J. Behav. Nutr. Phys. Act. 2013, 10, 1. [CrossRef]

74. Snijders, T.A.; Bosker, R.J. Multilevel Analysis: An Introduction to Basic and Advanced Multilevel Modeling; Sage: London, UK, 2012.

75. He, X.; Zhang, L. Quantifying the self-selection effect in residential location and vehicle use choices with a structural equation model. Transp. Res. Rec. 2014, 2453, 153-161. [CrossRef]

76. Kline, R.B. Principles and Practice of Structural Equation Modeling, 3rd ed.; Guilford Publications: New York, NY, USA, 2011.

77. Chung, J.H.; Kim, S.; Lee, Y.K.; Choi, Y.S. Multilevel structural equation model for activity participation and travel behavior: Data from the Puget sound transportation panel. Transp. Res. Rec. J. Transp. Res. Board 2004, 1898, 52-60. [CrossRef]

78. Kim, J.H.; Kim, H.D.; Chung, J.H. Weekend activity and travel behavior in a developing country: Empirical study using multilevel structural equation models. Transp. Res. Rec. J. Transp. Res. Board 2004, 1894, 99-108.

79. Brooke, S.; Ison, S.; Quddus, M. Analysing parking search ('cruising') time using generalised multilevel structural equation modelling. J. Transp. Econ. Policy (JTEP) 2018, 52, 202-220.

80. Kelloway, E.K. Using LISREL for Structural Equation Modeling: A Researcher's Guide; Sage: Thousand Oaks, CA, USA, 1998.

81. Heck, R.H. Multilevel modeling with SEM. In New Developments and Techniques in Structural Equation Modeling; Marcoulides, G.A., Schumacker, R.E., Eds.; Lawrence Erlbaum Associates, Inc.: Mahwah, NJ, USA, 2001; pp. 109-148.

82. Franke, G.R. Multicollinearity; Wiley International Encyclopedia of Marketing, John Wiley \& Sons, Inc.: Hoboken, NJ, USA, 2010; Volume 2. [CrossRef]

83. Kim, N.S.; Susilo, Y.O. Comparison of pedestrian trip generation models. J. Adv. Transp. 2013, 47, 399-412. [CrossRef]

84. Schauder, S.A.; Foley, M.C. The relationship between active transportation and health. J. Transp. Health 2015, 2, 343-349. [CrossRef]

85. Cao, X.; Mokhtarian, P.L.; Handy, S.L. Do changes in neighborhood characteristics lead to changes in travel behavior? A structural equations modeling approach. Transportation 2007, 34, 535-556. [CrossRef]

86. Stata Structural Equation Modeling Reference Manual Release 13. pp. 535-556. Available online: https://www.stata.com/manuals13/sem.pdf (accessed on 16 August 2020).

87. Roshan Zamir, K.; Nasri, A.; Baghaei, B.; Mahapatra, S.; Zhang, L. Effects of transit-oriented development on trip generation, distribution, and mode share in Washington, DC, and Baltimore, Maryland. Transp. Res. Rec. J. Transp. Res. Board 2014, 2413, 45-53. [CrossRef]

88. Mahmoudi, J.; Zhang, L. Impact of county-level built environment and regional accessibility on walking: A Washington, DC-Baltimore case study. J. Urban. Plan. Dev. 2018, 144, 04018020-1-04018020-15. [CrossRef]

89. Kline, R.B. Reverse arrow dynamics: Formative measurement and feedback loops. In Structural Equation Modeling: A Second Course; Hancock, G.R., Mueller, R.O., Eds.; Information Age Publishing Inc.: Greenwich, CT, USA, 2006; pp. 43-68.

Publisher's Note: MDPI stays neutral with regard to jurisdictional claims in published maps and institutional affiliations. 